\title{
Coverage and Handoff Analysis of 5G Fractal Small Cell Networks
}

\author{
Jiaqi Chen, Student Member, IEEE, Xiaohu Ge, Senior Member, IEEE, and Qiang Ni, Senior Member, IEEE
}

\begin{abstract}
It is anticipated that much higher network capacity will be achieved by the fifth generation (5G) small cell networks incorporated with the millimeter wave (mmWave) technology. However, mmWave signals are more sensitive to blockages than signals in lower frequency bands, which highlights the effect of anisotropic path loss in network coverage. According to the fractal characteristics of cellular coverage, a multi-directional path loss model is proposed for 5G small cell networks, where different directions are subject to different path loss exponents. Furthermore, the coverage probability, association probability, and the handoff probability are derived for 5G fractal small cell networks based on the proposed multi-directional path loss model. Numerical results indicate that the coverage probability with the multi-directional path loss model is less than that with the isotropic path loss model, and the association probability with long link distance, e.g., $150 \mathrm{~m}$, increases obviously with the increase of the effect of anisotropic path loss in $5 \mathrm{G}$ fractal small cell networks. Moreover, it is observed that the anisotropic propagation environment is having a profound impact on the handoff performance. Meanwhile, we could conclude that the resulting heavy handoff overhead is emerging as a new challenge for $5 \mathrm{G}$ fractal small cell networks.
\end{abstract}

Index Terms-Fractal small cell, anisotropic path loss, coverage probability, handoff.

\section{INTRODUCTION}

$\mathbf{O}$ $\mathrm{NE}$ of the primary goals of the fifth generation $(5 \mathrm{G})$ mobile communication is to provide $100 \mathrm{Mbps}$ to $1 \mathrm{Gbps}$ data rates anytime and anywhere. This goal can be achieved by combining a variety of $5 \mathrm{G}$ new technologies, such as massive multi-input multi-output (MIMO), millimeter wave (mmWave), and small cell networks [1], [2]. Base stations (BSs) equipped with large antenna arrays could achieve directivity gains through precoding/beamforming and could transmit mmWave signals, which results in high data rates. For example, the Samsung's prototype [3], which is a $4 \times 8$ uniform planar array working at $27.925 \mathrm{GHz}$, is able to offer excellent performance, where the reported peak data rate with no mobility is about $1 \mathrm{Gbps}$ over a range up to 1.7 kilometers in Line-of-Sight (LoS) transmissions or 200 meters in NonLine-of-Sight (NLoS) transmissions. The mmWave signals are more sensitive to blockages than signals in lower frequency bands due to the shorter wavelength [4], [5]. Thus, the link distance is reduced when the mmWave signals are transmitted,

Jiaqi Chen, Xiaohu Ge (Corresponding author) are with School of Electronic Information and Communications, Huazhong University of Science and Technology, Wuhan 430074, Hubei, P. R. China e-mail: chenjq_hust@mail.hust.edu.cn, xhge@mail.hust.edu.cn.

Qiang Ni is with InfoLab21, School of Computing and Communications, Lancaster University, Lancaster, LA1 4WA, UK, e-mail:q.ni@lancaster.ac.uk.

Manuscript received May 02, 2018; revised September 01, 2018, and December 02, 2018; accepted December 04, 2018. which results in the trend of small cells [6], [7]. In small cell networks, users frequently switch from a small cell BS (SBS) to another SBS since the coverage area of a small cell is significantly smaller than that of a macro cell. The frequent handoff issue in a small cell network with mmWave system becomes an essential factor that affects the network performance. On the other hand, the fractal coverage of small cells and the propagation environment have the critical influence on the handoff decision. Thus, it is vital to analyze the coverage and handoff performance of $5 \mathrm{G}$ small cell networks, considering the anisotropic mmWave signal attenuation in complex urban scenarios.

The coverage performance of small cell networks has been well studied. In small cell networks, multiple classes of BSs such as macro BSs, hotspot BSs, and femtocell BSs are incorporated to provide better coverage for a large number of users. Based on the stochastic geometry theory, the downlink and uplink coverage probabilities were derived for multi-tier heterogeneous cellular networks (HCNs) [8]. In the $\mathrm{HCN}$ with a dense small cell topology, the coverage probability for a typical user connecting to the strongest BS signal was analyzed in [9] by incorporating a flexible notion of BS load with a new idea of conditionally thinning the interference field, where penetrating analyses were extended to investigate the impact of network models on the $\mathrm{HCN}$ coverage performance. From the point of view of green communication, the coverage probability for a particular cell association scheme, such as the maximum received power association (MRPA) and the nearest base station association (NBA) schemes in the downlink of an $\mathrm{HCN}$, was derived aimed at establishing the fundamental limits on the achievable link and network energy efficiencies [10].

Moreover, transmission links could be distinguished as LoS vs. NLoS ones based on the differences in the path loss characteristic caused by blockages. Such differences become more influential in 5G mmWave systems than in the conventional network, and are generally taken into consideration when the performance of the mmWave system is analyzed. A comprehensive overview of mathematical models and analytical techniques for mmWave cellular systems were summarized in [11], where a baseline analytical approach based on stochastic geometry was presented which allows the computation of statistical distributions of the downlink signal-to-interferenceplus-noise-ratio (SINR). A better representation of the outage possibilities of mmWave communications was provided in [12], considering realistic path-loss and blockage models, which were derived from recently reported experimental data. In [12], simple and exact integrals as well as approximated and closed-form formulas for computing the coverage prob- 
ability and the average rate were obtained. The coverage and rate performance of mmWave cellular networks in major metropolitan cities like Manhattan and Chicago were studied in [13] to confirm that dense BS deployment is the key to achieve both better coverage and higher rate in mmWave cellular networks. Using a distance-dependent LoS probability function calculated by modeling the blockage distribution as a Poisson point process (PPP), a general framework was proposed in [14] to evaluate the coverage and rate performance in mmWave cellular networks. Only two types of transmission links, LoS and NLoS links are considered in the above analysis. In fact, the path loss exponents of different transmission links are entirely different due to the anisotropic propagation environment. In this case, an anisotropic path loss model should be provided to analyze the coverage performance of small cell networks with the mmWave system, compared with the distinction between LoS and NLoS transmission links.

Considering the more irregular coverage of mmWave cellular networks and smaller coverage area of a small cell, users have to switch from an SBS to another SBS frequently. An approach for calculating the handoff rate was proposed in [15] based on the angle mobility model. The handoff rate in mmWave $5 \mathrm{G}$ systems was investigated in [16], where the typical average handoff interval was shown to be in the range of several seconds. The handoff rate for a user was obtained in [17] for an irregular cellular network with the access point locations modeled as a homogeneous PPP. The vehicular handoff rate and the vehicular overhead ratio were utilized in [18] to evaluate the distance based vehicular mobility performance in 5G cooperative small cell networks. Efficient handoff algorithms were proposed in [19], [20], [21] to minimize the handoff and connection failure rates. In the most handoff analysis literature, the variety of the propagation environment during the user's moving is ignored in analyzing the handoff performance. However, due to the anisotropic propagation environment, changes in the path loss exponent of the transmission links during the user's moving have a significant influence on the handoff performance, especially in small cell networks with the mmWave system.

The research efforts in [6-18] were conducted with an assumption that the path loss is isotropic in a cellular scenario, where the most common path loss model is the uniform path loss model, i.e., the path loss exponent is a constant over the entire plane. A piecewise linear path loss model was proposed in [22] where different distance ranges are subject to different path loss exponents. Furthermore, researchers at New York University built a novel channel simulation software named NYUSIM based on the statistical spatial channel model for broadband mmWave wireless communication systems [23], [24]. In [23], the path loss exponents of LoS and NLoS transmissions were configured as different constants. However, our work in [25] indicated that the measured wireless cellular coverage boundary is irregular with respect to directions, and the statistical fractal characteristic of coverage boundary was proved to exist widely. Such a fractal characteristic is illustrated by the spectral density power-law behavior and the slowly decaying variances in the angle domain [25]. Moreover, in [26] the single cell coverage boundary was proved to bear a visible burst pattern, and the probability density function (PDF) of the single cell boundary was shown to have the heavy tail characteristic. In [27], the fractal coverage characteristic was first used to evaluate the performance of small cell networks. From the above result, it is evident that the propagation environment is anisotropic in a mmWave scenario. Although there exist a large amount of meaningful and essential studies for performance analysis of the small cell networks with mmWave, the impact of such anisotropic propagation environments is still not well investigated. Along with the prosperity of machine learning, rich learning schemes [28] obviously facilitate the research of the complex and accurate anisotropic path loss models.

In this paper, based on the fractal characteristics of cellular coverage [25], a multi-directional path loss model is proposed for $5 \mathrm{G}$ fractal small cell networks, where different directions are subject to different path loss exponents. Based on the proposed multi-directional path loss model, the coverage performance in SINR and rate terms are analyzed to estimate the impact of the anisotropic propagation environment. The handoff decision is made based on the association scheme and the channel state. Some existing analysis for the handoff performance works based on the assumption that the nearest neighbor SBS is the one with the highest SINR. However, the association state with the maximum SINR scheme is more complex in the anisotropic scenario than in the isotropic scenario. The association probability with the multi-directional path loss model is investigated to imply the influence of the anisotropic propagation environment and to lay the foundation for handoff analysis. Furthermore, the handoff performance of $5 \mathrm{G}$ fractal small cell networks is evaluated by the handoff probability and handoff rate. The handoff probability implies the performance of an individual handoff operation, and the handoff rate illustrates the overall network performance. The main contributions of this paper are as follows:

1) Based on the fractal characteristics of cellular coverage, a multi-directional path loss model is proposed to analyze the impact of an anisotropic propagation environment on the performance of $5 \mathrm{G}$ fractal small cell networks, where different directions are subject to different path loss exponents.

2) Based on the multi-directional path loss model, the coverage probability and the association probability concerning the desired link distance are derived to show that more complex propagation environments have negative impacts on the coverage performance of the $5 \mathrm{G}$ fractal small cell network. The assumption that the nearest base station for a user is the one with the highest SINR is typically not supported in 5G fractal small cell networks.

3) Based on the theoretical analysis on coverage probability and association probability of $5 \mathrm{G}$ fractal small cell networks, the impact of the anisotropic propagation environment on the handoff performance is first investigated by analyzing the handoff probability and handoff rate. Simulation results show that the anisotropic propagation environment has a profound effect on the handoff performance. 
The rest of the paper is organized as follows. Section II describes the system model. The coverage and association probability analyses are presented in Section III. The handoff probability and handoff rate are derived in Section IV. The simulation results are presented in Section V. Finally, conclusions are drawn in Section VI.

\section{SySTEM MODEL}

We consider a downlink small cell network in this paper. Without loss of generality, SBSs are assumed to be deployed randomly over an infinite plane $\mathbb{R}^{2}$ and modeled as a homogeneous PPP of intensity $\lambda$ [29]:

$$
\Phi=\left\{x_{i}, i=1,2,3, \ldots\right\},
$$

where $x_{i}$ is a Polar coordinate denoting the location of $\mathrm{SBS}_{i}$, i.e., $x_{i}=\left(r_{i}, \theta_{i}\right)$, with radial coordinate $r_{i}$ denoting the distance between $\mathrm{SBS}_{i}$ and the coordinate origin, and angular coordinate $\theta_{i}$. A typical user is assumed to be located at the coordinate origin, denoted as $u_{0}$.

Considering that the mmWave technology is excepted to be adopted in 5G small cell networks, a multi-directional path loss model is proposed in this section and aims at analyzing the impact of anisotropic propagation environment on the performance of mmWave small cell networks. What's more, the channel model is introduced.

\section{A. Multi-directional Path Loss Model}

With the emerging mmWave technology, researchers have examined the mmWave technology in some typical environments, e.g., an urban scenario with LoS transmissions, an urban scenario with NLoS transmissions, and the near-ground propagation in the forest. The empirical path loss exponents for the above three scenarios were reported as 2.1 [30], 3.19 [30], and 4 [31], respectively. It can be found that the path loss exponents are entirely different in these scenarios. Considering that the three typical propagation environments are irregularly distributed around SBSs, the links from a user to SBSs possibly pass through different environments such that the path loss exponents of these links are different. In this case, a uniform path loss model cannot be used to investigate the real anisotropic radio propagation. Thus, a multi-directional path loss model is proposed in this paper to evaluate the radio propagation in an anisotropic path loss scenario. A typical anisotropic path loss scenario is shown in Fig. 1a, where a user moves from point $\mathrm{A}$ to point $\mathrm{D}$ along the red line. The link between the user and a particular SBS passes through the wood when the user is in the range between A and B. The link between the user and the SBS passes through buildings when the user is in the range between $\mathrm{B}$ and $\mathrm{C}$. These links are NLoS. The link between the user and the SBS is LoS when the user is in the range between $\mathrm{C}$ and $\mathrm{D}$.

In this model, the coverage area centered at an SBS is equally partitioned into $M$ sections, and the path loss exponents of $M$ sections are assumed to be different, where the value of $M$ is artificially configured to model the anisotropic characteristic of a given propagation environment. When $M$ is large enough, the values of the path loss exponents in $M$ sections could

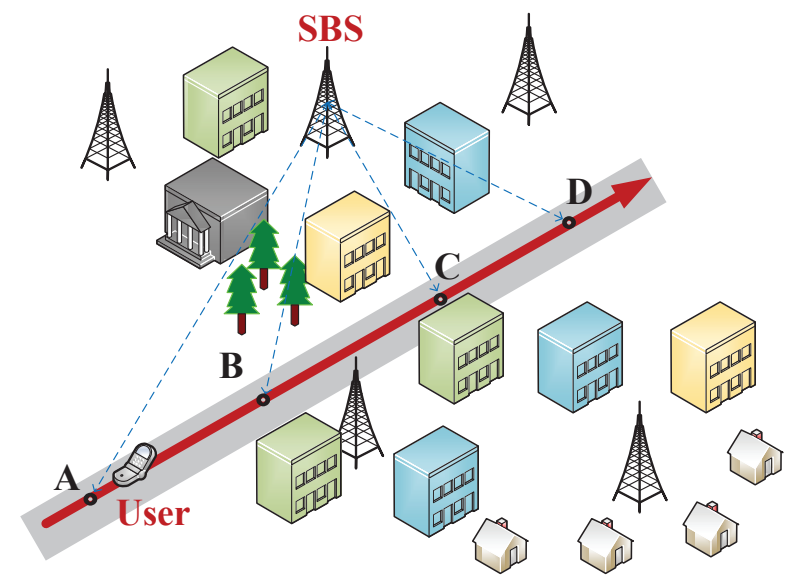

(a) A typical anisotropic propagation scenario.

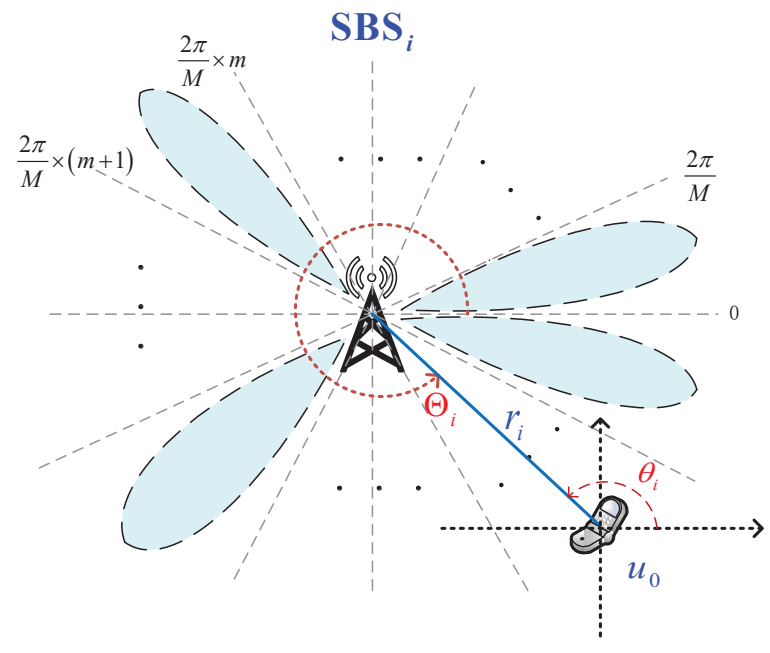

(b) The multi-directional path loss model.

Fig. 1. The typical anisotropic path loss scenario and model.

be regarded as the samples of the path loss exponent around the SBS. The sequence $\Omega_{i}=\left\{\alpha_{i m}\right\}$ denotes a stochastic process of the path loss exponent of $\mathrm{SBS}_{i}$, composed of the random variables $\alpha_{i m}$ with the index $m(m=1,2, \ldots, M)$. The anisotropic characteristic of the propagation environment could be analyzed with statistical methods based on these $M$ samples. Considering that the value of $M$ needs to be given to characterize the system performance, we show in Section V by simulation that the impact of $\mathrm{M}$ on the performance becomes stable when the value of $\mathrm{M}$ is larger than 400 .

The measured path loss exponent in real environments is mainly affected by the obstacle distribution, the atmospheric environment, the weather status, and diffraction and scattering effects. Since diffraction effects are negligible and there are only a few scattering clusters for mmWave [14], the impact of obstacle distribution dominates the path loss exponent in urban environments. The obstacles in real environments consisting of buildings, plants, cars, human bodies and so 
on, are independent [32], [33]. Considering that the sections in different directions are non-overlapping, the obstacles in different sections are independent. Therefore, in this paper the path loss exponent of the $m$-th section of $\operatorname{SBS}_{i}(i=1,2, \ldots)$, denoted as $\alpha_{i m}$, is assumed to be independently identically distributed (i.i.d.) random variables uniformly distributed with mean $\mu$ and variance $\sigma$, and its PDF is given as

$$
f\left(\alpha_{i m}\right)=\frac{1}{2 \sqrt{3} \sigma}, \alpha_{i m} \in[\mu-\sqrt{3} \sigma, \mu+\sqrt{3} \sigma] .
$$

Note that the path loss exponent of the $m$-th section at $\mathrm{SBS}_{i}$ is independent of the $m$-th section at $\operatorname{SBS}_{j}(j \neq i)$, i.e., $\alpha_{i m} \neq$ $\alpha_{j m}$.

A possible solution to obtain the path loss exponents of $M$ sections of an SBS in practice is introduced as follows. In order to let the SBS perceive the existence of the mobile phone at any time, the mobile phone must report the channel state information (CSI) back to the SBS regularly, or must immediately return when the SBS asks [34]. If the SBS is allowed to obtain the mobile phone's location, the path loss exponent can be estimated by a least square's method at the SBS based on the CSI and the location reported by the mobile phone. Considering the urban scenario, every SBS serves many users around it. The SBS collects the CSI and the locations of all online mobile phones, and then computes the path loss exponent of each section using the collected data from the section.

According to the 3GPP path loss models summarized in TR 38.900 [30], the path loss exponent in UMi-Street Canyon scenario is 2.1 when the link distance is smaller than the breakpoint distance, and that becomes 4 when the link distance is larger than the breakpoint distance. The characteristic mutations of path loss exponent occur at the breakpoint. Considering a mmWave system in the small cell network that $f_{c}=24 \mathrm{GHz}$, the height of SBS is $5 \mathrm{~m}$, the height of user is $1.5 \mathrm{~m}$, the breakpoint distance is calculated as $640 \mathrm{~m}$, which comfortably exceeds the average coverage radius of an SBS given as $\frac{1}{\sqrt{\pi \lambda}} \approx 56 \mathrm{~m}$. We consider the $5 \mathrm{G}$ fractal small cell networks with high density in which the link distances are usually smaller than the breakpoint distance. In this case, the difference in path loss exponents of different directions is mainly considered in this paper. The multi-directional path loss over the link between $\mathrm{SBS}_{i}$ and the typical user $u_{0}$ is set as

$$
L\left(x_{i}\right)=r_{i}^{-\alpha_{i m}},
$$

when $\frac{2 \pi}{M}(m-1) \leq \Theta_{i}<\frac{2 \pi}{M} m(m=1, \ldots, M)$, where $\Theta_{i}$ is the angle of the vector from $\mathrm{SBS}_{i}$ to $u_{0}$, which is calculated to locate the section that the user stays in, shown in Fig. $1 b$. The angle $\Theta_{i}$ is given as

$$
\Theta_{i}=\left\{\begin{array}{ll}
\theta_{i}+\pi & \theta_{i} \in[0, \pi) \\
\theta_{i}-\pi & \theta_{i} \in[\pi, 2 \pi)
\end{array} .\right.
$$

Such a multi-directional path loss model can be degraded into the isotropic path loss model with the uniform path loss exponent over the entire plane when $\sigma=0$, i.e., $\alpha_{i 1}=\alpha_{i 2}=\cdots=\alpha_{i M}=\alpha_{c}=\mu$ as a constant with

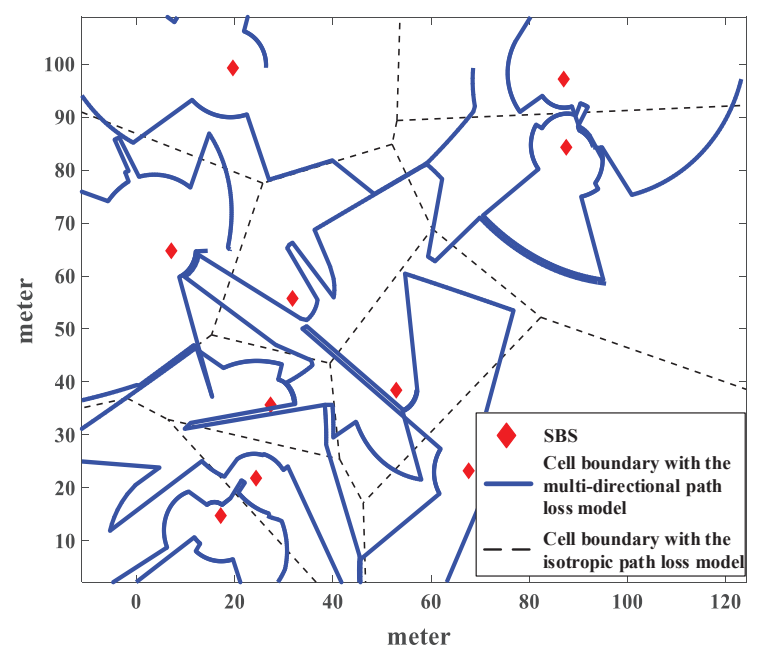

Fig. 2. Illustration of $5 \mathrm{G}$ fractal small cell network structure with the multidirectional path loss model $(M=5, \mu=4, \sigma=1)$, compared with the isotropic path loss model.

probability 1 . The isotropic path loss model could be expressed as $L^{1}\left(x_{i}\right)=r_{i}^{-\mu}$.

To illustrate the $5 \mathrm{G}$ fractal small cell network structure, assume that a user is associated with the SBS from which the user receives the maximum SINR, and users only suffer the path loss during propagation. Every cell is assumed to include only one SBS. The locations at which the user receive equal SINRs from the two adjacent SBSs, make up the cell boundaries. For the multi-directional path loss model, the blue solid lines in Fig. 2 depict cell boundaries inside which a polygon corresponds a cell coverage. For the traditional isotropic path loss model, the SBS with the maximum SINR is the closest SBS for the user. In this case, the cell boundary can be obtained through the Delaunay Triangulation method [35] expressed as the black dashed lines in Fig. 2.

What is more, the carrier frequency factor $f_{c}$ in the path loss, $P L=32.4+20 \lg (r)+20 \lg \left(f_{c}\right)(\mathrm{dB})$ [30], is normalized to simplify the analysis of the impact of the anisotropic path loss, considering that the same carrier frequency is used at all SBSs.

\section{B. Channel Model}

When the channel fading of each transmission link is assumed to be governed by the i.i.d. Rayleigh fading, the received signal power at $u_{0}$ from $\mathrm{SBS}_{i}$ is given as

$$
P_{i}=P_{T} G_{i} h_{i} L\left(x_{i}\right),
$$

where $h_{i}$ denotes the power gain from Rayleigh fading between $u_{0}$ and $\mathrm{SBS}_{i}$, which is assumed to be governed by an exponential distribution with mean 1 similar to [11]. For simplicity, but without loss of generality, all SBSs are assumed to transmit with the same transmission power $P_{T}$. $G_{i}$ denotes the antenna gain obtained from the $\mathrm{SBS}_{i}$ and $u_{0}$.

For tractability of the analysis, the actual array patterns are approximated by a sectored antenna model. Both the user and 
TABLE I

THE PROBABILITY DISTRIBUTION OF ANTENNA GAIN $G_{i}$

\begin{tabular}{|c|c|c|c|c|}
\hline Value & $g_{1}=M_{t} M_{r}$ & $g_{2}=M_{t} m_{r}$ & $g_{3}=m_{t} M_{r}$ & $g_{4}=m_{t} m_{r}$ \\
\hline Probability & $p_{1}=\frac{\phi_{t}}{2 \pi} \frac{\phi_{r}}{2 \pi}$ & $p_{2}=\frac{\phi_{t}}{2 \pi}\left(1-\frac{\phi_{r}}{2 \pi}\right)$ & $p_{3}=\left(1-\frac{\phi_{t}}{2 \pi}\right) \frac{\phi_{r}}{2 \pi}$ & $p_{4}=\left(1-\frac{\phi_{t}}{2 \pi}\right)\left(1-\frac{\phi_{r}}{2 \pi}\right)$ \\
\hline
\end{tabular}

its serving SBS will estimate the channels including angles of arrivals and fading gains, and then adjust their antenna steering orientations accordingly to exploit the maximum antenna gain. Errors in channel estimation are neglected, and so are errors in time and carrier frequency synchronization in this paper. Thus, $M_{t}$ and $M_{r}$ are the main lobe gains of the SBS and user, respectively; $m_{t}$ and $m_{r}$ are the side lobe gains of the SBS and user, respectively; $\phi_{t}$ and $\phi_{r}$ denote the main lobe width and side lobe width of the SBS and user, respectively. The antenna gain of the desired link is $M_{t} M_{r}$, and the antenna gain of the interfering link is a random variable with the distribution shown in Table I [14].

In order to obtain the best channel condition, a user is configured to be associated with a particular SBS based on the SINR value, i.e., when the SINR value of the link between the user and this SBS is the maximum. The SBS associated with the typical user $u_{0}$ is denoted as $\mathrm{SBS}_{k}$, where

$$
k=\underset{x_{i} \in \Phi}{\operatorname{argmax}} \operatorname{SINR}\left(x_{i}\right),
$$

with

$$
\begin{aligned}
\operatorname{SINR}\left(x_{i}\right) & =\frac{P_{i}}{I\left(x_{i}\right)+\sigma_{n}^{2}} \\
& =\frac{P_{T} M_{t} M_{r} h_{i} r_{i}^{-\alpha_{i}}}{\sum_{x_{j} \in \Phi, j \neq i} P_{T} G_{j} h_{j} r_{j}^{-\alpha_{j}}+\sigma_{n}^{2}},
\end{aligned}
$$

which is the SINR value of the link between $u_{0}$ and $\mathrm{SBS}_{i}$ with $\sigma_{n}^{2}$ denoting the noise power and $I\left(x_{i}\right)$ denoting the aggregate interference. The path loss exponents $\alpha_{i}$ and $\alpha_{j}$ in (7) depend on the angles $\Theta_{i}$ and $\Theta_{j}$ of the vectors from $\mathrm{SBS}_{i}$ and $\mathrm{SBS}_{j}$ to the typical user, respectively.

\section{Coverage And Association Probability}

In this section, the coverage and association probabilities of $5 \mathrm{G}$ fractal small cell networks are derived based on the multi-directional path loss model. Numerical analyses of the coverage and association probabilities are conducted to evaluate the performance impacted by the anisotropic propagation environment.

\section{A. Coverage Probability}

We analyze the coverage performance with the multidirectional path loss model of $5 \mathrm{G}$ fractal small cell networks in SINR and rate terms. The expressions for the SINR and rate coverage probability with the multi-directional path loss model are derived. The SINR coverage probability to the point of view of the typical user $u_{0}$ is the probability that the desired received SINR at $u_{0}$ is larger than a threshold $\tau$. Considering the maximum SINR association, the SINR coverage probability is given as

$$
P_{C}(\tau)=\operatorname{Pr}\left\{\max _{x_{i} \in \Phi} \operatorname{SINR}\left(x_{i}\right)>\tau\right\} .
$$

Lemma 1. Based on the Lemma 1 in [36], when the SINR threshold $\tau>\frac{M_{t} M_{r}}{m_{t} m_{r}}$, at most one SINR can be greater than $\tau$.

Proof: See Appendix A.

Theorem 1. The SINR coverage probability for the typical user at the origin is given as

$$
\begin{aligned}
& P_{C}(\tau)=\frac{\lambda \pi}{\sqrt{3} \sigma} \int_{0}^{\infty} \int_{\mu-\sqrt{3} \sigma}^{\mu+\sqrt{3} \sigma} r \exp \left(-\frac{\lambda \pi^{2}}{\sqrt{3} \sigma} \sum_{l=1}^{4} p_{l}\right. \\
& \left.\times \int_{\mu-\sqrt{3} \sigma}^{\mu+\sqrt{3} \sigma} \frac{\left(\frac{\tau r^{\alpha_{i}} g_{l}}{M_{t} M_{r}}\right)^{\frac{2}{\alpha}}}{\alpha} \csc \left(\frac{2 \pi}{\alpha}\right) d \alpha-\frac{\tau r^{\alpha_{i}} \sigma_{n}^{2}}{P_{T} M_{t} M_{r}}\right) d \alpha_{i} d r .
\end{aligned}
$$

Proof: See Appendix B.

Furthermore, we analyze the distribution of the achievable rate $R_{0}$ of $5 \mathrm{G}$ fractal small cell networks with the multidirectional path loss model. The achievable rate is defined as

$$
R_{0}=B_{W} \log _{2}\left(1+\max _{x_{i} \in \Phi} \operatorname{SINR}\left(x_{i}\right)\right),
$$

where $B_{W}$ is the bandwidth assigned to the typical user $u_{0}$. The rate coverage probability is the probability that the achievable rate of the typical user is larger than the threshold $\gamma$, which is expressed as

$$
\begin{aligned}
P_{R}(\gamma) & =\operatorname{Pr}\left\{R_{0}>\gamma\right\} \\
& =\operatorname{Pr}\left\{B_{W} \log _{2}\left(1+\max _{x_{i} \in \Phi} \operatorname{SINR}\left(x_{i}\right)\right)>\gamma\right\} \\
& =\operatorname{Pr}\left\{\max _{x_{i} \in \Phi} \operatorname{SINR}\left(x_{i}\right)>2^{\frac{\gamma}{B_{W}}}-1\right\} \\
& =P_{C}\left(2^{\frac{\gamma}{B_{W}}}-1\right) .
\end{aligned}
$$

To express the impact of the multi-directional path loss model on the coverage performance of the $5 \mathrm{G}$ fractal small cell network, numerical results of the SINR and rate coverage probabilities are presented considering the factors including the SINR threshold $\tau$, the rate threshold $\gamma$, the SBS density $\lambda$, and the mean $\mu$ and the variance $\sigma$ of the path loss exponent. The default parameters are set as: $P_{T}=30 \mathrm{dBm}, B_{W}=$ $500 \mathrm{MHz}, \sigma_{n}^{2}=-174 \mathrm{dBm} / \mathrm{MHz} \times 500 \mathrm{MHz}=-87 \mathrm{dBm}$ [37], $M_{t}=M_{r}=10 \mathrm{~dB}, m_{t}=m_{r}=0 \mathrm{~dB}, \phi_{t}=7^{\circ}, \phi_{r}=60^{\circ}$ [3], $\lambda=10^{-4}, \tau=20 \mathrm{~dB}$ and $\mu=4$ in this paper unless noted otherwise. The large variance $\sigma$ implies the significant difference among the path loss exponents of SBSs in the $5 \mathrm{G}$ fractal small cell network. 


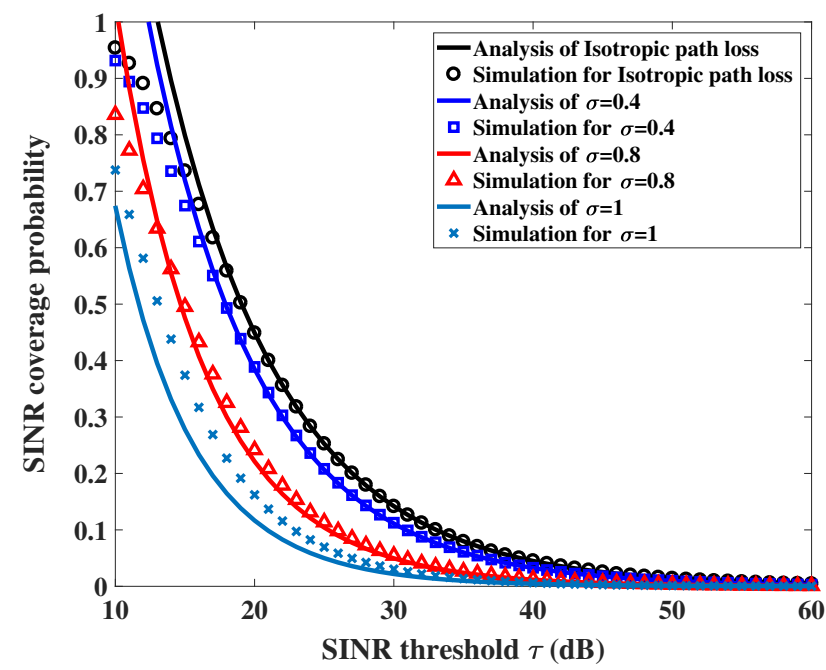

Fig. 3. The SINR coverage probability with respect to the SINR threshold $\tau$, considering different variances of the path loss exponent.

Fig. 3 illustrates the SINR coverage probability with respect to the SINR threshold $\tau$, considering different variances $\sigma$ of the path loss exponent. The SINR coverage probability decreases with the increase of the SINR threshold. When the variance is equal to zero, i.e., $\sigma=0$, the path loss exponent is a constant with probability 1 , and is equal to the mean $\mu$. Thus, the case with $\sigma=0$ represents the isotropic path loss model in this paper. When the SINR threshold is fixed, the SINR coverage probability decreases with the increase of the variance $\sigma$, which indicates that the anisotropic path loss model has a negative influence on the coverage performance of the small cell network. According to the proof of Lemma 1, the result shows numerically that this SINR coverage probability model holds very accurately.

Fig. 4 shows the SINR coverage probability with respect to the variance of the path loss exponent $\sigma$, considering different mean values $\mu$. The larger mean value $\mu$ results in that the SINR coverage probability increases, which implies that the coverage performance is better in the higher path loss attenuation environment due to the more severe attenuation of the interference signal. When the mean $\mu$ is fixed, the SINR coverage probability decreases with the increase of the variance $\sigma$, which shows that the coverage performance is better in the more stable propagation environment, especially in the isotropic propagation environment with the case $\sigma=0$. It is because the path loss exponents of a part of interference links are so small in the anisotropic propagation environment that increase the aggregate interference power compared to the isotropic path loss model.

Fig. 5 illustrates the rate coverage probability with respect to the achievable rate threshold $\gamma$, considering different antenna gains. The rate coverage probability increases with the larger ratio of the main lobe gain to the side lobe gain $\frac{M_{t}}{m_{t}}$. It can be found in the figure that the red and black lines with $\frac{M_{t}}{m_{t}}=10 \mathrm{~dB}-0 \mathrm{~dB}=5 \mathrm{~dB}-(-5) \mathrm{dB}=10 \mathrm{~dB}$ ate below the green and blue lines with $\frac{M_{t}}{m_{t}}=20 \mathrm{~dB}-0 \mathrm{~dB}=$ $10 \mathrm{~dB}-(-10) \mathrm{dB}=20 \mathrm{~dB}$. What's more, the rate coverage

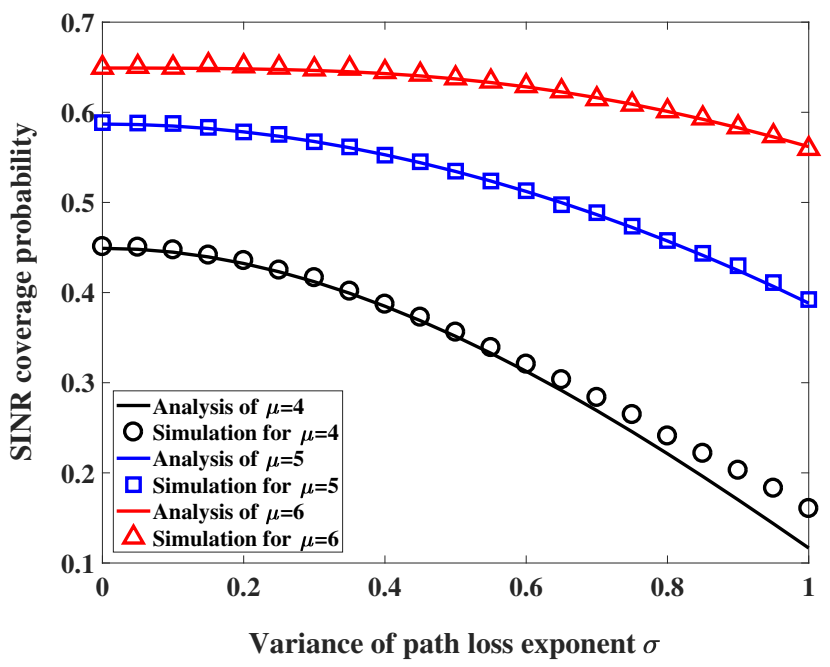

Fig. 4. The SINR coverage probability with respect to the variance of the path loss exponent $\sigma$, considering different mean values $\mu$.

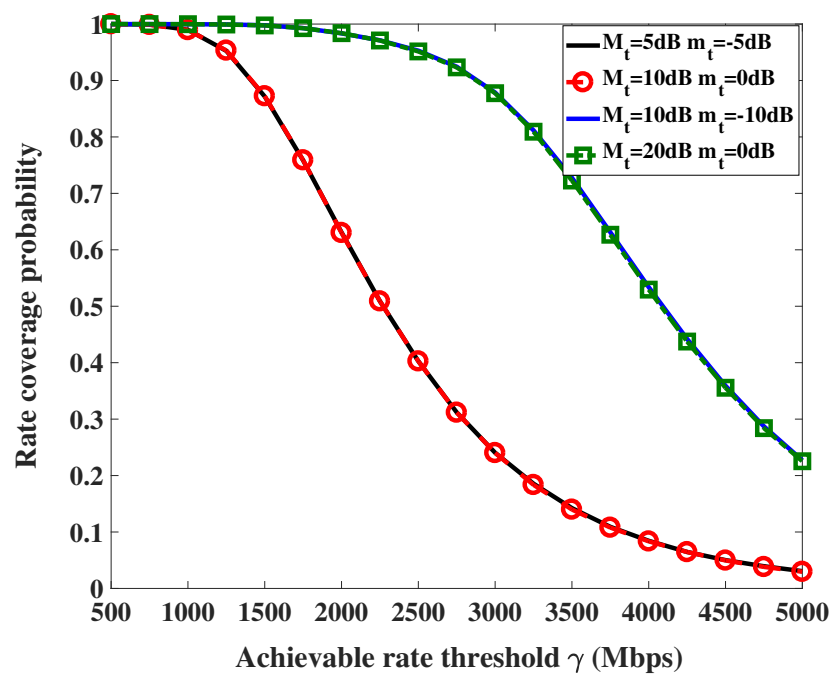

Fig. 5. The rate coverage probability with respect to the achievable rate threshold $\gamma$, considering different antenna gains.

probability is independent from the values of and when the ratio of the main lobe gain to the side lobe gain $\frac{M_{t}}{m_{t}}$ is fixed.

\section{B. Association Probability}

Some existing analysis for the handoff performance works based on the assumption that the nearest neighbor SBS to be the one with the highest SINR. When the isotropic path loss model is adopted, it can be proved that a user is covered by the nearest SBS with the maximum probability. However, the association probability with an LoS SBS may be greater than that with an NLoS SBS even when the distance between the LoS SBS and the user is larger than the distance between the NLoS SBS and the user in a complex propagation environment, especially in small cell networks [39]. In this section, association probabilities for the typical user with the different SBSs in 5G fractal small cell networks are analyzed based on the multi-directional path loss model to address the above situations. 
In this paper, a user is assumed to be associated with the SBS that leads to the maximum received SINR. The association probability with respect to the distance $r$ between $u_{0}$ and the desired SBS $\mathrm{SBS}^{r}$ is expressed as

$$
\begin{gathered}
P_{A}(r)=\operatorname{Pr}\left\{\operatorname{SINR}(r)=\max _{x_{i} \in \Phi} \operatorname{SINR}\left(x_{i}\right)\right\}, \\
\operatorname{SINR}(r)=\frac{P_{T} M_{t} M_{r} h_{r} r^{-\alpha_{r}}}{I(r)+\sigma_{n}^{2}},
\end{gathered}
$$

where $h_{r}$, and $\alpha_{r}$ denote the antenna gain, power gain from Rayleigh fading, and path loss exponent of the link between $u_{0}$ and the desired SBS SBS ${ }^{r}$, respectively. $I(r)$ is the received interference of the user $u_{0}$.

Considering the difficulty of calculating the association probability due to the complex expression of SINR, an approximation that greatly simplifies the analysis is that the user is associated with the SBS that leads to the maximum received power. The following Lemma shows that the approximation has the high accuracy, and the accuracy increases with the narrower main lobes at the transmitter and receiver.

Lemma 2. The probability that $\operatorname{SINR}\left(x_{k}\right)$ is the maximum is at least $\left(1-\frac{\phi_{t}}{360}\right)\left(1-\frac{\phi_{r}}{360}\right)$ when the corresponding received signal power $P_{k}$ is maximum.

Proof: See Appendix C.

Note that the system level simulations are given for the probability in Lemma 2, expressed as $\operatorname{Pr}\{\operatorname{SINR}(r) \mid P(r)\}$. The results are shown in Table II and imply that the probability is higher when the narrower main lobes are equipped at the transmitter and receiver. In this case, the probability that $\operatorname{SINR}(r)$ is the maximum can be approximated by the probability that $P(r)$ is the maximum. Based on Lemma 2, the association probability is further expressed as

$$
P_{A}(r) \approx \operatorname{Pr}\left\{P(r)=\max _{x_{i} \in \Phi} P_{i}\right\},
$$

where $P(r)$ denotes the received signal power at $u_{0}$ from $\mathrm{SBS}^{r}$, expressed as $P(r)=G_{r} h_{r} r^{-\alpha_{r}}$.

Theorem 2. The association probability with respect to the distance $r$ between $u_{0}$ and the desired SBS SBS ${ }^{r}$ is approximately given as

$$
\begin{aligned}
& P_{A}(r)=\sum_{m=1}^{4} \frac{p_{m}}{2 \sqrt{3} \sigma} \int_{0}^{\infty} \int_{\mu-\sqrt{3} \sigma}^{\mu+\sqrt{3} \sigma} \exp \left(-h_{r}-\frac{\lambda \pi}{\sqrt{3} \sigma} \sum_{n=1}^{4} p_{n}\right. \\
& \left.\times \int_{\mu-\sqrt{3} \sigma}^{\mu+\sqrt{3} \sigma} \frac{\left(\frac{g_{m}}{g_{n}} h_{r} r^{-\alpha_{r}}\right)^{-\frac{2}{\alpha_{i}}} \Gamma\left(\frac{2}{\alpha_{i}}\right)}{\alpha_{i}} d \alpha_{i}\right) d \alpha_{r} d h_{r} .
\end{aligned}
$$

Proof: See Appendix D.

Fig. 6a shows the association probability with respect to the distance $r$ between $u_{0}$ and the desired SBS SBS ${ }^{r}$, considering different variances $\sigma$. When the variance of the path loss exponent is fixed, the association probability decreases with the increase of the distance $r$ between $u_{0}$ and the desired SBS SBS ${ }^{r}$. When the distance $r$ is smaller than 80 meters, the association probability increases with the decrease of the variance $\sigma$. When the distance $r$ is larger than 160 meters, the association probability increases with the increase of the variance $\sigma$. The realistic association of users in small cell networks is more complicated than the NBA scheme. The result also implies that the nearest base station association scheme is not the desired method to obtain the best channel gain in small cell networks with mmWave. Thus, we need to pay more attention to the novel association scheme when analyzing the performance of small cell networks with mmWave. Fig. $6 \mathrm{~b}$ shows the association probability with respect to the distance $r$ between $u_{0}$ and the desired SBS SBS ${ }^{r}$, considering different intensities of SBSs $\lambda$. The variance of the path loss exponent is set as $\sigma=0.8$. When the distance $r$ is fixed, the association probability decreases with the increase of the intensity of SBSs. What's more, we now focus on the approximation in Lemma 2 by comparing the theoretical and simulated results for association probability in Fig. 6. As expected, the simulated and analytical results match reasonably well.

Fig. 7 shows the association probability with respect to the variances $\sigma$, considering different distances $r$ between $u_{0}$ and $\mathrm{SBS}^{r}$. The mean value of the path loss exponent in this figure is equal to 5 to ensure that the minimum value of the path loss exponent is larger than 2. When the distance $r$ between $u_{0}$ and the desired SBS SBS ${ }^{r}$ is shorter than $90 \mathrm{~m}$, the association probability decreased with the increase of the variance $\sigma$ of the path loss exponent. When the distance is larger than $110 \mathrm{~m}$, the association probability has a peak value at particular variances.

\section{HANdofF PERformance}

In particular, the handoff is crucial for keeping users connected while moving around. Frequent handoff in small cell networks affects not only service quality of users but also network performance, such as throughput and energy efficiency. Conditional handoff analysis is based on the isotropic propagation environment. However, the negative influence of the anisotropic environment on the handoff performance is predictable in future small cell networks. In this section, the handoff probability and handoff rate are derived based on the user random walk mobility model to investigate the impact of the multi-directional path loss model.

\section{A. Handoff Probability}

Assumed that the serving SBS of the typical user $u_{0}$ at the location $l_{0}$ is denoted as $\mathrm{SBS}^{r}$, i.e., the SINR of $\mathrm{SBS}^{r}$ at the location $l_{0}$ is the maximum. Let $l_{0}$ and $l_{1}$ respectively denote the locations at the start and end of a short time movement, and the moving time is the time interval between the end of the last handoff decision and the begin of the next one. 3GPP Standard defines six handoff events for cellular networks [40]. In this paper, the analysis of handoff performance with the multidirectional path loss model focuses on the most common Event A2. The Event A2 occurs when the SINR of the serving SBS becomes smaller than a threshold $\tau_{h}$, and the trigger condition can be expressed as

$$
\operatorname{SINR}(\tilde{r})<\tau_{h}-\tau_{\text {hys }},
$$


TABLE II

THE PROBAbiLITy that SINR $(r)$ IS THE MAXIMUM WHEN $P(r)$ IS THE MAXIMUM $(r=50 \mathrm{~m})$.

\begin{tabular}{|c|c|c|c|c|c|c|}
\hline$\phi_{t}$ & $7^{\circ}$ & $20^{\circ}$ & $60^{\circ}$ & $60^{\circ}$ & $20^{\circ}$ & $7^{\circ}$ \\
\hline$\phi_{r}$ & $60^{\circ}$ & $60^{\circ}$ & $60^{\circ}$ & $20^{\circ}$ & $10^{\circ}$ & $10^{\circ}$ \\
\hline \hline $\operatorname{Pr}\{$ SINR $(r) \mid P(r)\}$ & 0.9176 & 0.9035 & 0.8822 & 0.9036 & 0.9553 & 0.9709 \\
\hline
\end{tabular}

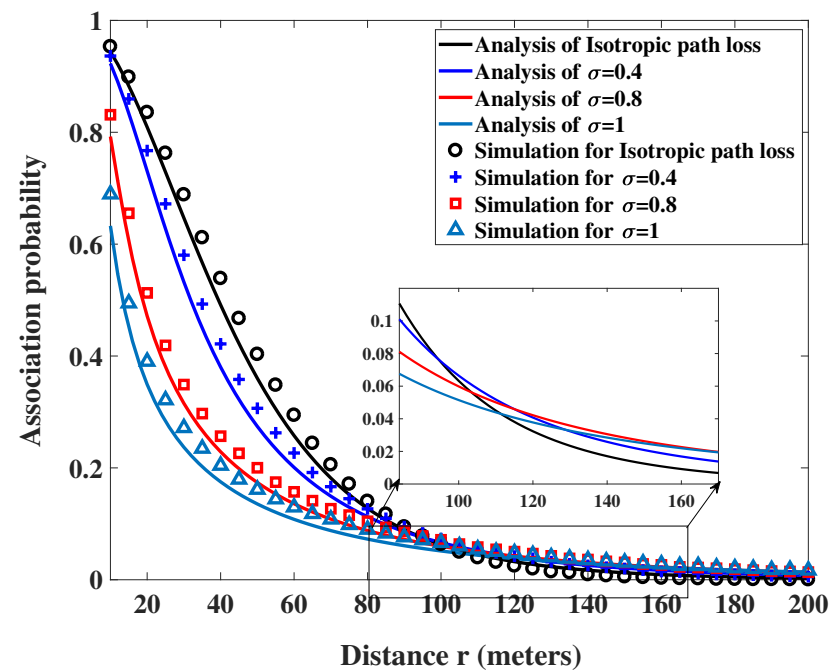

(a)

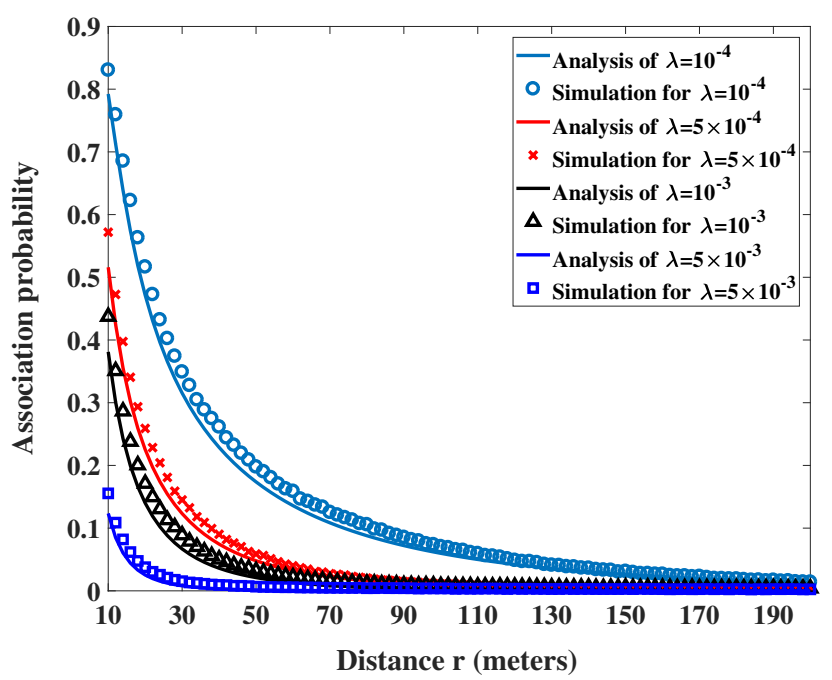

(b)

Fig. 6. The association probability with respect to the distance $r$ between $u_{0}$ and the desired SBS $\mathrm{SBS}^{r}$, considering different variances of the path loss exponent and the intensities of SBSs.

where $\tau_{\text {hys }}$ is a hysteresis parameter added for reducing redundant handoffs (e.g. ping-pong effect), and $\tilde{r}$ is the distance between the serving SBS and the user at the new location $l_{1}$. Once inequality (16) is satisfied for the user, an Event A2 handoff is triggered, and the user needs to select a suitable target SBS. In this case, the handoff probability, $P_{H}(v t)$, denotes the probability that a handoff occurs when the user moves a distance $v t$, which is expressed as $P_{H}(v t)=\int_{0}^{\infty} \operatorname{Pr}\{\mathrm{A} 2\} d r$. When the distance between the serving SBS and the user at location $l_{0}$ is $r$, the probability that the Event $\mathrm{A} 2$ occurs is

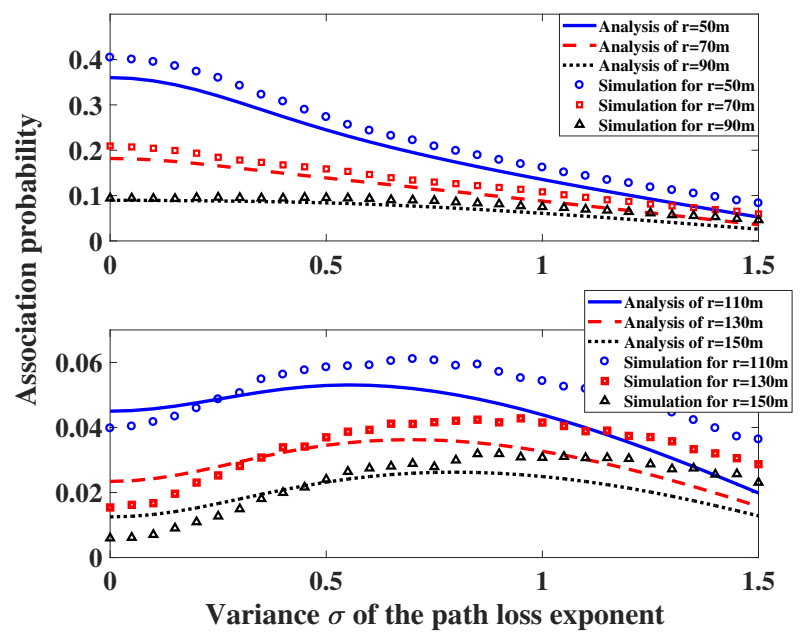

Fig. 7. The association probability with respect to the variances $\sigma$, considering different distances $r$ between $u_{0}$ and $\mathrm{SBS}^{r}$.

given as

$$
\operatorname{Pr}\{\mathrm{A} 2\}=\operatorname{Pr}\left\{\operatorname{SINR}(\tilde{r})<\tau_{h}-\tau_{\text {hys }}\right\} .
$$

Before calculating the handoff probability, the relationship between the locations $l_{0}$ and $l_{1}$ are considered. The movement of a user is assumed to be governed by a random walk mobility model in this paper. The user moves from its original location to a new location by randomly choosing a direction $\varphi$ and a speed $v$, where the speed and direction are both chosen from pre-defined ranges $\left[v_{\min }, v_{\max }\right]$ and $[0,2 \pi)$, respectively. Such a random walk mobility model is shown in Fig. 8a, where $u_{0}$ moves from the location $l_{0}$ to $l_{1}, \Theta_{i}$ is the angle between the horizontal line and the line crossing through $l_{0}$ and $\mathrm{SBS}_{i}, \tilde{\Theta}_{i}$ is the angle between the horizontal line and the line crossing through $l_{1}$ and $\mathrm{SBS}_{i}, r_{i}$ and $\tilde{r}_{i}$ are the distances between $\mathrm{SBS}_{i}$ and $l_{0}, l_{1}$, respectively, $t$ is the user moving time, and $v t$ is the distance of the user moving with the speed $v$ and time $t$. The relationship between the locations $l_{0}$ and $l_{1}$ is expressed as

$$
\begin{gathered}
\tilde{r}_{i}^{2}=r_{i}^{2}+(v t)^{2}+2 v t r_{i} \cos \left(\varphi-\Theta_{i}\right), \\
\tilde{\Theta}_{i}=\Theta_{i}+\arctan \left(\frac{v t \sin \left(\varphi-\Theta_{i}\right)}{r+v t \cos \left(\varphi-\Theta_{i}\right)}\right) .
\end{gathered}
$$

Two special cases are considered in the derivation. One case is that the user moves away from the serving SBS: $\mathrm{SBS}^{r}$, i.e., $\varphi=\Theta^{r}$, where $\Theta^{r}$ is the angle between the horizontal line and the line crossing through $l_{0}$ and $\mathrm{SBS}^{r}$. The other case is that the user moves to the direction perpendicular to the line crossing through $l_{0}$ and $\mathrm{SBS}^{r}$, i.e., $\varphi=\Theta^{r}+\frac{\pi}{2}$. 


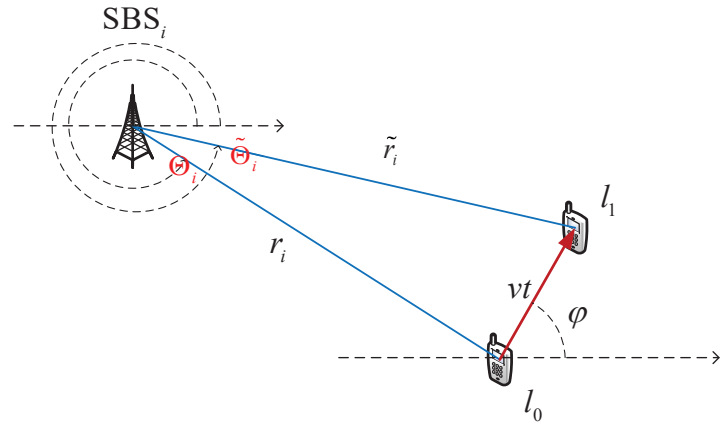

(a) The random walk mobility model.

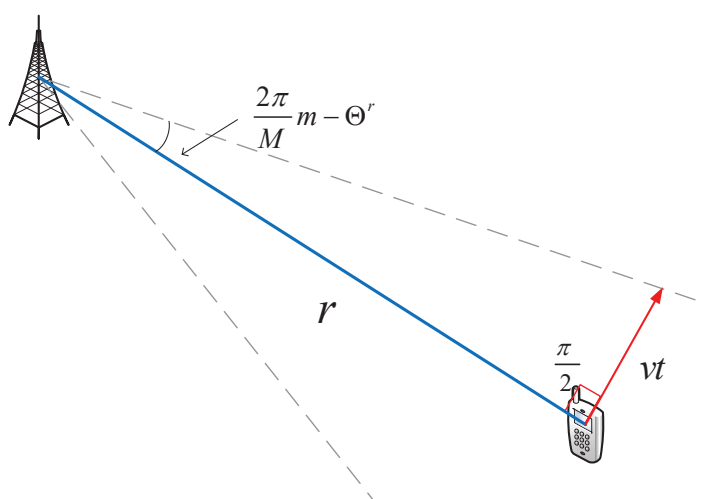

m-th section

(b) The scenario that user moves at the direction $\varphi=\Theta^{r}+\frac{\pi}{2}$.

Fig. 8. The random walk mobility model with the multi-directional path loss model.

Case 1: $\varphi=\Theta^{r}$. In this case, the path loss exponent of the link between the user and the serving SBS is unchanged. When the user moves a distance $v t$, the received SINR of the serving SBS at the user is given as

$$
\operatorname{SINR}^{0}(\tilde{r})=\frac{P_{T} M_{t} M_{r} \tilde{h}_{r}(r+v t)^{-\alpha_{r}}}{I(r+v t)+\sigma_{n}^{2}},
$$

where $\tilde{h}_{r}$ is the power gain from Rayleigh fading at the new location, which is independent of $h_{r}$.

The handoff probability is expressed as

$P_{H}^{0}(v t)=\int_{r} \mathbb{E}_{\alpha_{r}}\left[\operatorname{Pr}\left\{\operatorname{SINR}^{0}(\tilde{r})<\tau_{h}-\tau_{\text {hys }} \mid \alpha_{r}\right\} P_{A}\left(r \mid \alpha_{r}\right)\right] d r$,

with the conditional association probability following (15)

$$
\begin{gathered}
P_{A}\left(r \mid \alpha_{r}\right)=\sum_{m=1}^{4} p_{m} \int_{0}^{\infty} \exp \left(-h_{r}-\frac{\lambda \pi}{\sqrt{3} \sigma} \sum_{n=1}^{4} p_{n}\right. \\
\left.\times \int_{\mu-\sqrt{3} \sigma}^{\mu+\sqrt{3} \sigma} \frac{\left(\frac{g_{m}}{g_{n}} h_{r} r^{-\alpha_{r}}\right)^{-\frac{2}{\alpha_{i}}} \Gamma\left(\frac{2}{\alpha_{i}}\right)}{\alpha_{i}} d \alpha_{i}\right) d h_{r} .
\end{gathered}
$$

Theorem 3. When the user moves to the direction $\varphi=\Theta^{r}$, the handoff probability is

$$
\begin{aligned}
& P_{H}^{0}(v t) \\
& =\int_{0}^{\infty} \int_{\mu-\sqrt{3} \sigma}^{\mu+\sqrt{3} \sigma}\left(1-\exp \left(-\left(\frac{\tau_{h}-\tau_{h y s}}{P_{T} M_{t} M_{r}}\right) \sigma_{n}^{2}(r+v t)^{\alpha_{r}}\right)\right. \\
& \left.\times \exp \left(-\frac{\lambda \pi^{2}}{\sqrt{3} \sigma} \sum_{l=1}^{4} p_{l} \int_{\alpha} \frac{\left(\frac{\left(\tau_{h}-\tau_{h y s}\right)(r+v t)^{\alpha_{r}} g_{l}}{M_{t} M_{r}}\right)^{\frac{2}{\alpha}} \csc \left(\frac{2 \pi}{\alpha}\right)}{\alpha} d \alpha\right)\right) \\
& \times\left(\sum _ { m = 1 } ^ { 4 } p _ { m } \int _ { 0 } ^ { \infty } \operatorname { e x p } \left(-h_{r}-\frac{\lambda \pi}{\sqrt{3} \sigma} \sum_{n=1}^{4} p_{n}\right.\right. \\
& \left.\left.\times \int_{\mu-\sqrt{3} \sigma}^{\mu+\sqrt{3} \sigma\left(\frac{g_{m}}{g_{n}} h_{r} r^{-\alpha_{r}}\right)^{-\frac{2}{\alpha_{i}}} \Gamma\left(\frac{2}{\alpha_{i}}\right)} \alpha_{i}\right) d \alpha_{r}\right) d \alpha_{r} d r .
\end{aligned}
$$

Proof: See Appendix E.

Case 2: $\varphi=\Theta^{r}+\frac{\pi}{2}$. As shown in Fig. 8b, the path loss exponent is unchanged when the moving distance satisfies $v t \leq r \tan \left(\frac{2 \pi}{M} m-\Theta^{r}\right)$. When the moving distance is larger than $r \tan \left(\frac{2 \pi}{M} m-\Theta^{r}\right)$, the path loss exponent changes. Then, the received SINR of the serving SBS at the user is given as

$$
\operatorname{SINR}^{90}(\tilde{r})=\frac{P_{T} M_{t} M_{r} \tilde{h}_{r}\left(r^{2}+(v t)^{2}\right)^{-\frac{\tilde{\alpha}_{r}}{2}}}{I\left(\sqrt{r^{2}+(v t)^{2}}\right)+\sigma_{n}^{2}},
$$

where $\tilde{\alpha}_{r}$ denotes the new path loss exponent of the link between the serving SBS and the user located at sections except for the $m$-th section. Given that the distance $r$ between the user and the serving SBS is fixed and known, angle $\Theta^{r}$ is assumed to be uniformly distributed in $[0,2 \pi)$ and independent of the distance $r$ [41]. In this case, $\Delta \Theta=\frac{2 \pi}{M} m-\Theta^{r}$ is uniformly distributed in $\left[0, \frac{2 \pi}{M}\right)$. The handoff probability is expressed as

$$
\begin{aligned}
& P_{H}^{90}(v t) \\
& =\frac{M}{2 \pi} \int_{0}^{\frac{2 \pi}{M}}\left(\int_{0}^{\frac{v t}{\tan (\Delta \Theta)}} \operatorname{Pr}\left\{\operatorname{SINR}^{90}(\tilde{r})<\tau_{h}-\tau_{\text {hys }}\right\} P_{A}(r) d r\right. \\
& +\int_{\frac{v t}{\tan (\Delta \Theta)}}^{\infty} \mathbb{E}_{\alpha_{r}}\left[\operatorname{Pr}\left\{\operatorname{SINR}^{90}(\tilde{r})<\tau_{h}-\tau_{\text {hys }} \mid \tilde{\alpha}_{r}=\alpha_{r}\right\}\right. \\
& \left.\left.\times P_{A}\left(r \mid \alpha_{r}\right)\right] d r\right) d \Delta \Theta .
\end{aligned}
$$

The detailed calculation is similar to the Theorem 3, which is omitted here. The final expression of the handoff probability with $\varphi=\Theta^{r}+\frac{\pi}{2}$ is not shown here.

\section{B. Handoff Rate}

To evaluate the effect of the anisotropic path loss on the handoff performance, the handoff overhead should be taken into consideration for 5G fractal small cell networks. During handoff execution, a user releases the serving SBS and establishes a new association with the target SBS through control protocols with the two related SBSs. The handoff overhead is contributed by control signals transmitted between the user 
and the SBSs. Hence, the overall handoff overhead is directly proportional to the handoff rates.

To easily evaluate the handoff overhead, the handoff rate is defined as the average number of handoffs per unit time. According to the handoff control protocols [42], the handoff decision procedures are periodically executed, where the signal detection and information exchanging between the user and the original associated SBS are conducted in each given period. Such a period is denoted as the detection interval $t_{d}$. Thus, in this paper Monte-Carlo simulations are utilized to analyze the handoff rate of $5 \mathrm{G}$ fractal small cell networks, with the multi-directional path loss model.

We consider a small cell network deployed in an urban area, and the network consists of a user and a varying number of SBSs. The user is initially located at the central of a circular area with radius equal to $1000 \mathrm{~m}$, and SBSs are randomly distributed in the area. Some parameters related to system model are the same as those in Section III. The user moves to a random direction at a speed $v$. In each period $t_{d}$, the channel state information is reported to the serving SBS by the user, and a handoff decision mechanism is executed. We assume that a perfect initial cell search can be performed, and thus the user can discover SBSs correctly when a handoff occurs. The number of handoffs in 1000 seconds is recorded to calculate the handoff rate.

\section{SIMULATION RESULTS}

The handoff probability and handoff rate are analyzed for $5 \mathrm{G}$ fractal small cell networks with the multi-directional path loss model (where the uniform path loss model is treated as a special case with $\sigma=0$ ) in this section. The default parameters are configured as in Section III. The SINR threshold for handoff with a hysteresis parameter is configured as $\tau_{h}-\tau_{\text {hys }}=0 \mathrm{~dB}$.

Fig. 9 shows the handoff probability with respect to the moving distance $v t$ in the two special cases, considering different variances of the path loss exponent. In this figure, the number of sections of each SBS is configured as $M=3$. The handoff probability increases with the increase of the moving distance in a detection interval. And the handoff probability increases with the increase of the variance of the path loss exponent. The difference between the handoff probabilities in the case 1 and case 2 could be ignored at the same condition with moving distance and variance of the path loss exponent. The results imply that the direction of the user movement has no influence on the handoff performance.

Fig. 10 illustrates the handoff rate with respect to the variance $\sigma$ of the path loss exponent, considering different values of $M$. For the analysis of the handoff rate, the detection interval $t_{d}$ is configured as 1 second, and the user moving speed $v$ is $5 \mathrm{~m} / \mathrm{s}$. The handoff rate increases with increase of the variance $\sigma$ when the value of $M$ is fixed. The slope of the curve with $M=100$, is much larger than slopes of the other curves, i.e., the handoff rate with larger $M$ is growing more than the handoff rate with small $M$. The result shows that the complex anisotropic propagation environment has negative impact on the handoff performance, and the handoff

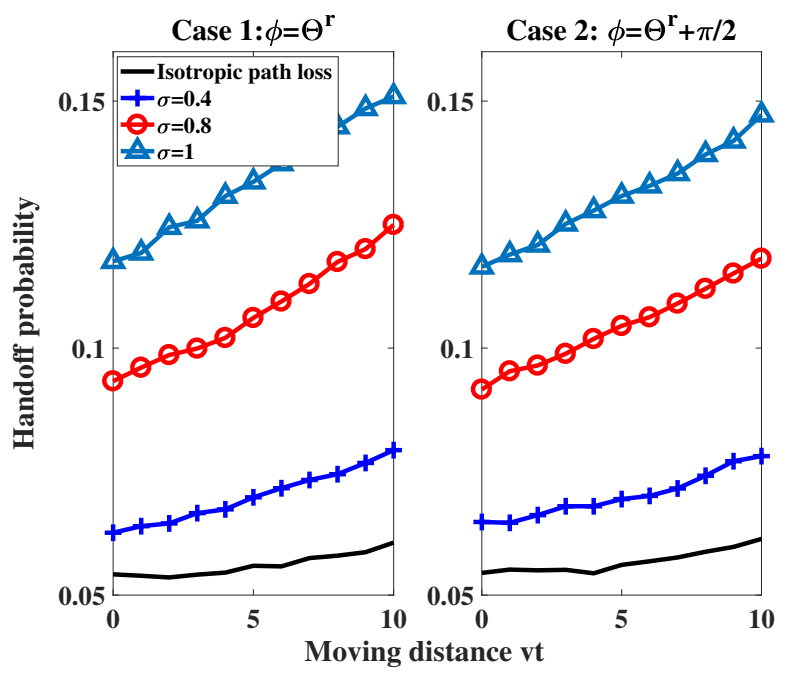

Fig. 9. The handoff probability with respect to the moving distance $v t$ in the two special cases, considering different variances of the path loss exponent.

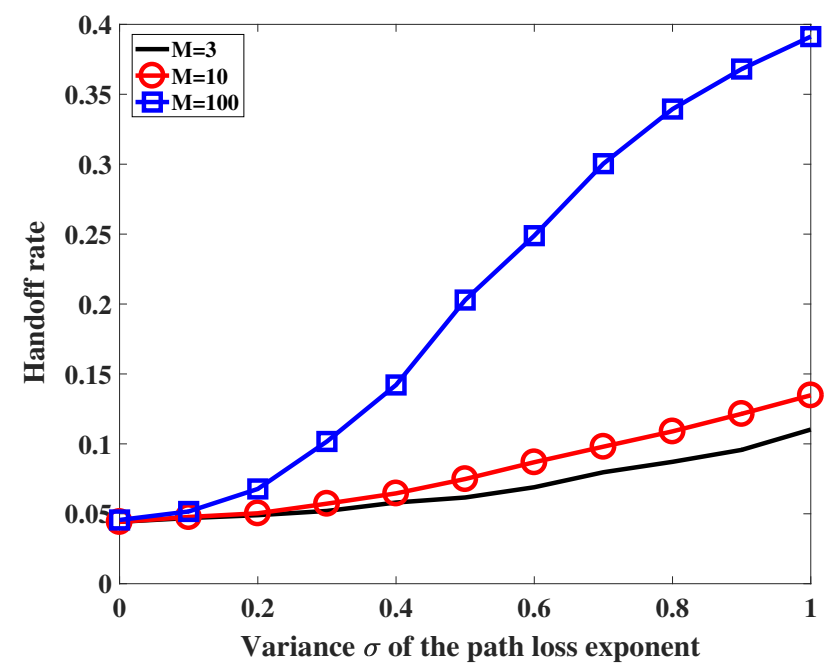

Fig. 10. The handoff rate with respect to the variance $\sigma$ of the path loss exponent, considering different values of $M$.

probability analyzed based on the isotropic path loss model and the actual handoff probability have a huge difference in more complex anisotropic propagation environment.

Fig. 11 shows the handoff rate with respect to $M$, considering different variances $\sigma$ of the path loss exponent. The handoff rate increases with the increase of $M$, the number of sections of each SBS. In Fig. 11a, the value of $M$ is in the range of $[10,100]$, and the handoff rate increases rapidly with the increase of $M$. In Fig. 11b, the value of $\mathrm{M}$ is in the range of $[100,1000]$, the increase of the handoff rate becomes slowdown when $M$ is larger than 400. The impact of $M$ on the handoff rate becomes stable when $M$ is larger than 400 which advises that the coverage region of an SBS can be equally partitioned into 400 sections in practice.

In Fig. $11 \mathrm{~b}$ the handoff rate with the multi-directional path loss model has a upper limit which increases with the increase of the variance. It is because the probability that the path loss exponent is unchanged during the movement from $l_{0}$ to 


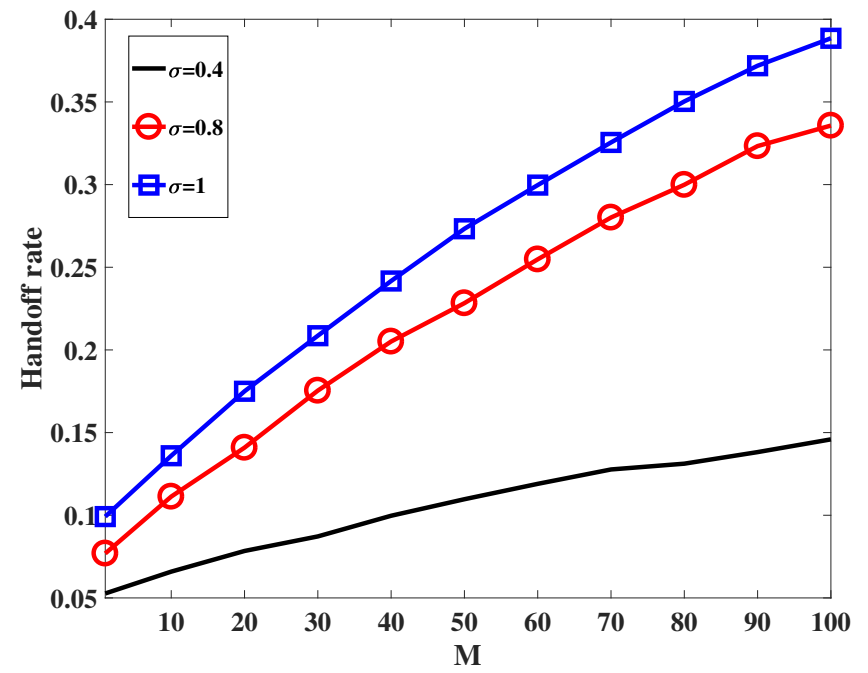

(a)

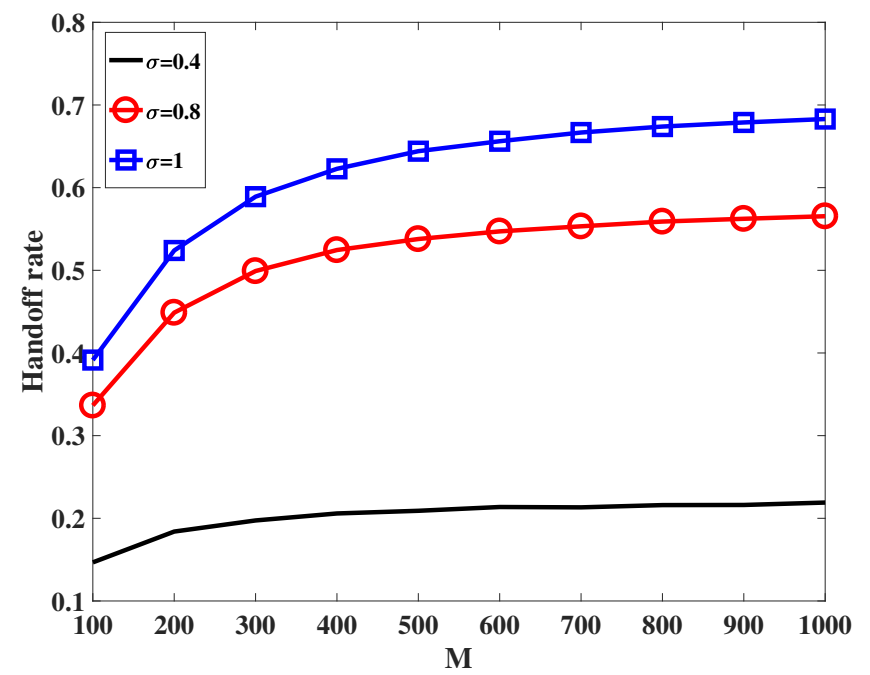

(b)

Fig. 11. The handoff rate with respect to $M$, considering different variances $\sigma$ of the path loss exponent.

$l_{1}$ is zero due to $\frac{2 \pi}{M}$ approaching zero when $M$ approaches infinity. In this case, the path loss exponent of the link at each detection time is an independent random variable. The limit of the handoff rate of $5 \mathrm{G}$ fractal small cell networks is influenced by the distribution of the path loss exponent, not influenced by the value of $M$. And the increase percentage of the handoff rate with the large variance over that with low variance is more than $100 \%$ when the large variance is twice as much as the low variance.

\section{Conclusions}

The small cell network is a promising technology for $5 \mathrm{G}$ mobile communication systems to increase the network capacity. Considering the complex environment in urban scenarios, the anisotropic path loss effect cannot be ignored any more. A multi-directional path loss model was proposed to analyze the impact of the anisotropic path loss exponent on performance in $5 \mathrm{G}$ fractal small cell networks. The new coverage probability, association probability and handoff probability were derived based on the proposed multi-directional path loss model. Numerical results indicate that the association probability with short link distance, e.g., $50 \mathrm{~m}$, decreases obviously with the increase of the effect of anisotropic path loss in $5 \mathrm{G}$ fractal small cell networks. Moreover, it was observed that the handoff probability with the multi-directional path loss model is larger than the handoff probability with the isotropic path loss model. When the coverage area of each SBS is equally partitioned into 400 sections, the handoff performance can be stably analyzed with the multi-directional path loss model. When the multi-directional path loss model with specific parameters for a special scenario is obtained, the association and handoff probability can be calculated directly based on the statistic characteristic of the measured parameters, which can provide straightforward guidance for the deployment of wireless communication networks.

\section{APPENDIX A \\ PROOF OF LEMMA 1}

Proof: Let SINR $\left(x_{i}\right)$ be denoted as $b_{i}$, and $a_{i}$ represent $P_{T} G_{i} h_{i} r_{i}^{-\alpha_{i}}$. We have

$$
\begin{aligned}
& b_{i}=\frac{\frac{M_{t} M_{r} a_{i}}{G_{i}}}{\sum_{j \neq i} a_{j}+\sigma_{n}^{2}}=\frac{\frac{M_{t} M_{r} a_{i}}{G_{i}}}{\sum_{\Phi} a_{j}+\sigma_{n}^{2}-a_{i}} \\
& \Longrightarrow \frac{a_{i}}{\sum_{\Phi} a_{j}+\sigma_{n}^{2}}=\frac{1}{1+\frac{M_{t} M_{r}}{G_{i} b_{i}}} \\
& \Longrightarrow \sum_{\Phi} \frac{1}{1+\frac{M_{t} M_{r}}{G_{i} b_{i}}}+\frac{\sigma_{n}^{2}}{\sum_{\Phi} a_{j}+\sigma_{n}^{2}}=1 .
\end{aligned}
$$

Then following the proof of Lemma 1 in [36], (26) could be satisfied if only one of the $b_{i}\left(i \in \mathbb{R}^{+}\right)$is greater than $\frac{M_{t} M_{r}}{m_{t} m_{r}}$, i.e., $\frac{M_{t} M_{r}}{G_{i} b_{i}}<1$, and $\frac{1}{1+\frac{M_{t} M_{r}}{G_{i} b_{i}}}>\frac{1}{2}$, because the value of $G_{i}$ is always greater or equal to $m_{t} m_{r}$. Assumed that two $b_{i}$ are greater than $\frac{M_{t} M_{r}}{m_{t} m_{r}}$, denoting as $b_{1}, b_{2}$. Therefore, $\frac{1}{1+\frac{M M_{t} M_{r}}{G_{1} b_{1}}}>\frac{1}{2}, \frac{1}{1+\frac{M t_{t} M_{r}}{G_{2} b_{2}}}>\frac{1}{2}$, and $\frac{1}{1+\frac{M_{t} M_{r}}{G_{1} b_{1}}}+\frac{1}{1+\frac{M_{t} M_{r}}{G_{2} b_{2}}}>1$ which is in contradiction with (26). This contradictory situation occurs when more $b_{i}$ are greater than $\frac{M_{t} M_{r}}{m_{t} m_{r}}$. In this case, at most one $b_{i}$ can be greater than $\frac{M_{t} M_{r}}{m_{t} m_{r}}$, and Lemma 1 is completely proved.

\section{APPENDIX B PROOF OF THEOREM 1}

Proof: The coverage probability in a 5G fractal small cell network with the maximum SINR association can be expressed as

$$
\begin{aligned}
P_{C}(\tau) & =\operatorname{Pr}\left\{\max _{x_{i} \in \Phi} \operatorname{SINR}\left(x_{i}\right)>\tau\right\} \\
& =\operatorname{Pr}\left\{\bigcup_{x_{i} \in \Phi} \frac{P_{T} M_{t} M_{r} h_{i} r_{i}^{-\alpha_{i}}}{I\left(x_{i}\right)+\sigma_{n}^{2}}>\tau\right\} .
\end{aligned}
$$


Furthermore, according to Lemma 1, when the SINR threshold $\tau>\frac{M_{t} M_{r}}{m_{t} m_{r}}$, the coverage probability is derived as

$$
\begin{aligned}
P_{C}(\tau) & =\mathbb{E}\left[\mathbf{1}\left\{\bigcup_{x_{i} \in \Phi} \operatorname{SINR}\left(x_{i}\right)>\tau\right\}\right] \\
& =\mathbb{E}\left[\sum_{x_{i} \in \Phi} \mathbf{1}\left\{\operatorname{SINR}\left(x_{i}\right)>\tau\right\}\right] \\
& \stackrel{(a)}{=} \lambda \int_{\mathbb{R}^{2}} \operatorname{Pr}\left\{\frac{P_{T} M_{t} M_{r} h_{i} r_{i}^{-\alpha_{i}}}{I\left(x_{i}\right)+\sigma_{n}^{2}}>\tau\right\} d x_{i} \\
& =\lambda \int_{\mathbb{R}^{2}} \operatorname{Pr}\left\{h_{i}>\frac{\tau r_{i}^{\alpha_{i}}\left(I\left(x_{i}\right)+\sigma_{n}^{2}\right)}{P_{T} M_{t} M_{r}}\right\} d x_{i} \\
& \stackrel{(b)}{=} \lambda \int_{\mathbb{R}^{2}} \mathbb{E}_{\alpha_{i}}\left[\mathcal{L}_{I}\left(\frac{\tau r_{i}^{\alpha_{i}}}{P_{T} M_{t} M_{r}}\right) e^{-\frac{\tau r_{i}^{\alpha_{i}} \sigma_{n}^{2}}{P_{T} M_{t} M_{r}}}\right] d x_{i},
\end{aligned}
$$

where $\mathbb{E}_{X}[\cdot]$ denotes the statistical average with respect to the random variable $X, \mathbf{1}\{\cdot\}$ denotes the indicator function, step (a) follows from Campbell Mecke Theorem [38], and step (b) follows from the assumption that the power gain of the channel fading $h_{i}$ is the exponential distribution with mean 1 and the definition of Laplace transform. $\mathcal{L}_{I}(\cdot)$ is the Laplace functional of the aggregated interference power $I\left(x_{i}\right)$.

Considering the aggregated interference power expressed as $I\left(x_{i}\right)=\sum_{x_{j} \in \Phi, j \neq i} P_{T} G_{j} h_{j} r_{j}^{-\alpha_{j}}$, the Laplace function of $I\left(x_{i}\right)$ is further derived as

$$
\begin{aligned}
& \mathcal{L}_{I}(s)=\mathbb{E}\left[e^{\left.-s \sum_{x_{j} \in \Phi, j \neq i} P_{T} G_{j} h_{j} r_{j}^{-\alpha_{j}}\right]}\right] \\
& =\mathbb{E}_{\Phi}\left[\prod_{x_{j} \in \Phi, j \neq i} \mathbb{E}_{h_{j}, \alpha_{j}, G_{j}}\left[e^{\left.-s P_{T} G_{j} h_{j} r_{j}^{-\alpha_{j}}\right]}\right]\right] \\
& \stackrel{(c)}{=} \exp \left(-\lambda \int_{\mathbb{R}^{2}}\left(1-\mathbb{E}_{h, \alpha, G}\left[e^{-s P_{T} G h|x|^{-\alpha}}\right]\right) d x\right) \\
& \stackrel{(d)}{=} \exp \left(-\frac{\lambda}{2 \sqrt{3} \sigma} \int_{\mathbb{R}^{2}} \int_{\alpha} \sum_{l=1}^{4} p_{l}\right. \\
& \left.\times\left(1-\frac{1}{1+s P_{T} g_{l}|x|^{-\alpha}}\right) d \alpha d x\right),
\end{aligned}
$$

where step (c) follows from probability generating functional (PGFL) of the PPP [38], which states that $\mathbb{E}_{\Phi}\left[\prod_{x \in \Phi} f(x)\right]=$ $\exp \left(-\lambda \int_{\mathbb{R}^{2}}(1-f(x)) d x\right)$ for $f(x)<1$, step (d) is the result of statistical averages with respect to the antenna gain $G$, the power gain $h$ and the path loss exponent $\alpha$ with the assumption on the uniform distribution of the path loss exponent. Let $t=\frac{|x|^{\alpha}}{s P_{T} g_{l}}$, we have

$$
\begin{aligned}
& \mathcal{L}_{I}(s)=\exp \left(-\frac{\lambda \pi}{\sqrt{3} \sigma} \sum_{l=1}^{4} p_{l} \int_{\alpha} \frac{\left(s P_{T} g_{l}\right)^{\frac{2}{\alpha}}}{\alpha} \int_{0}^{\infty} \frac{t^{\frac{2}{\alpha}-1}}{1+t} d t d \alpha\right) \\
& =\exp \left(-\frac{\lambda \pi^{2}}{\sqrt{3} \sigma} \sum_{l=1}^{4} p_{l} \int_{\mu-\sqrt{3} \sigma}^{\mu+\sqrt{3} \sigma} \frac{\left(s P_{T} g_{l}\right)^{\frac{2}{\alpha}}}{\alpha} \csc \left(\frac{2 \pi}{\alpha}\right) d \alpha\right) .
\end{aligned}
$$

Let $s=\frac{\tau r_{i}^{\alpha_{i}}}{P_{T} M_{t} M_{r}}$ in (30), the coverage probability is given as (9), which completes the proof.

\section{APPENDIX C \\ PROOF OF LEMMA 2}

Proof: Let $P$ denote the event that the received signal power $P_{k}$ is the maximum, and let $S$ denote the event that $\mathrm{SINR}_{k}$ is the maximum. According to the derivation in (26) in Appendix A, let $a_{i}=P_{i}$ (the positive values, not in $\mathrm{dB}$ ) and $b_{i}=$ $\operatorname{SINR}\left(x_{i}\right)$, we have

$$
\frac{a_{k}}{\sum_{\Phi} a_{j}+\sigma_{n}^{2}}=\frac{1}{1+\frac{M_{t} M_{r}}{G_{k} b_{k}}}
$$

The event $P$ is that $a_{k}$ is the maximum, equivalent to that $G_{k} b_{k}$ is the maximum based on (31), which is expressed as

$$
G_{k} b_{k}>G_{i} b_{i} \Rightarrow \frac{b_{k}}{b_{i}}>\frac{G_{i}}{G_{k}} x_{i} \in \Phi, i \neq k
$$

The event $S$ is that $b_{k}$ is the maximum, equivalent to that $\frac{b_{k}}{b_{i}} \geq 1$ for $x_{i} \in \Phi, i \neq k$.

When the event $P$ is true, the probability that the event $S$ is true is expressed as

$$
\operatorname{Pr}\{S \mid P\}=\operatorname{Pr}\left\{b_{k}=\max _{x_{i} \in \Phi} b_{i} \mid a_{k}=\max _{x_{i} \in \Phi} a_{i}\right\} .
$$

When $\frac{G_{i}}{G_{k}} \geq 1, \frac{b_{k}}{b_{i}}>\frac{G_{i}}{G_{k}} \geq 1$ denoting that the event $S$ is true. When $\frac{G_{i}}{G_{k}}<1, b_{k} \geq b_{i}$ and $b_{k}<b_{i}$ could be true possibly. Moreover, let the values of the antenna gain be arranged as the descending order, $g_{1} \geq g_{2} \geq g_{3} \geq g_{4}$, the conditional probability that the event $S$ is true is further given as

$$
\begin{aligned}
& \operatorname{Pr}\{S \mid P\} \geq \operatorname{Pr}\left\{\frac{G_{i}}{G_{k}} \geq 1, \forall x_{i} \in \Phi, i \neq k\right\} \\
& =\operatorname{Pr}\left\{G_{k}=g_{1}, G_{i}=g_{1}(i \neq k)\right\} \\
& +\operatorname{Pr}\left\{G_{k}=g_{2}, G_{i} \in\left\{g_{1}, g_{2}\right\}(i \neq k)\right\} \\
& +\operatorname{Pr}\left\{G_{k}=g_{3}, G_{i} \in\left\{g_{1}, g_{2}, g_{3}\right\}(i \neq k)\right\}+\operatorname{Pr}\left\{G_{k}=g_{4}\right\} \\
& =p_{4}+p_{3} \prod_{i \neq k}\left(p_{1}+p_{2}+p_{3}\right)+p_{2} \prod_{i \neq k}\left(p_{1}+p_{2}\right)+p_{1} \prod_{i \neq k} p_{1} \\
& \stackrel{(a)}{=} p_{4}=\left(1-\frac{\phi_{t}}{360}\right)\left(1-\frac{\phi_{r}}{360}\right)
\end{aligned}
$$

where step (a) is obtained because the three continuous multiplications are equal to zero when the number of SBSs is infinite.

\section{APPENDIX D \\ PROOF OF THEOREM 2}

Proof: The association probability with respect to the distance $r$ between $u_{0}$ and the desired SBS $\operatorname{SBS}^{r}$ is expressed 
as

$$
\begin{aligned}
& P_{A}(r)=\operatorname{Pr}\left\{\operatorname{SINR}(r)=\max _{x_{i} \in \Phi} \operatorname{SINR}\left(x_{i}\right)\right\} \\
& \approx \operatorname{Pr}\left\{G_{r} h_{r} r^{-\alpha_{r}}=\max _{x_{i} \in \Phi} G_{i} h_{i} r_{i}^{-\alpha_{i}}\right\} \\
& =\mathbb{E}\left[\prod_{x_{i} \in \Phi} \mathbb{E}_{G_{i}, \alpha_{i}}\left[\operatorname{Pr}\left\{G_{r} h_{r} r^{-\alpha_{r}} \geq G_{i} h_{i} r_{i}^{-\alpha_{i}}\right\}\right]\right] \\
& =\mathbb{E}\left[\exp \left(-\lambda \int_{\mathbb{R}^{2}} 1-\mathbb{E}_{G_{i}, \alpha_{i}}\left[\operatorname{Pr}\left\{h_{i} \leq \frac{G_{r} h_{r} r^{-\alpha_{r}} x^{\alpha_{i}}}{G_{i}}\right\}\right] d x\right)\right] \\
& =\mathbb{E}\left[\exp \left(-\lambda \int_{\mathbb{R}^{2}} \mathbb{E}_{G_{i}, \alpha_{i}}\left[\exp \left(-\frac{G_{r} h_{r} r^{-\alpha_{r}} x^{\alpha_{i}}}{G_{i}}\right)\right] d x\right)\right], \quad(35)
\end{aligned}
$$

where $G_{r}, h_{r}$, and $\alpha_{r}$ denote the antenna gain, power gain from Rayleigh fading, and path loss exponent of the link between $u_{0}$ and the desired SBS SBS ${ }^{r}$, respectively. Using the PDFs of the power gain from Rayleigh fading, path loss exponent, and the probability distribution of antenna gain, the association probability is further expressed as (15), which completes the proof.

\section{APPENDIX E}

\section{PROOF OF THEOREM 3}

Proof: The probability $\operatorname{Pr}\left\{\operatorname{SINR}^{0}(\tilde{r})<\tau_{h}-\tau_{\text {hys }} \mid \alpha_{r}\right\}$ is derived as

$$
\begin{aligned}
& \operatorname{Pr}\left\{\operatorname{SINR}^{0}(\tilde{r})<\tau_{h}-\tau_{\text {hys }} \mid \alpha_{r}\right\} \\
& =\mathbb{E}\left[\operatorname{Pr}\left\{\tilde{h}_{r}<\left(\frac{\tau_{h}-\tau_{\text {hys }}}{P_{T} M_{t} M_{r}}\right)\left(I(r+v t)+\sigma_{n}^{2}\right)(r+v t)^{\alpha_{r}}\right\}\right] \\
& \stackrel{(a)}{=} 1-\mathbb{E}\left[\exp \left(-\left(\frac{\tau_{h}-\tau_{\text {hys }}}{P_{T} M_{t} M_{r}}\right)\left(I(r+v t)+\sigma_{n}^{2}\right)(r+v t)^{\alpha_{r}}\right)\right] \\
& =1-\exp \left(-\left(\frac{\tau_{h}-\tau_{\text {hys }}}{P_{T} M_{t} M_{r}}\right) \sigma_{n}^{2}(r+v t)^{\alpha_{r}}\right) \\
& \times \mathcal{L}_{I}\left(\left(\frac{\tau_{h}-\tau_{\text {hys }}}{P_{T} M_{t} M_{r}}\right)(r+v t)^{\alpha_{r}}\right),
\end{aligned}
$$

where (a) is obtained by the PDF of the power gain $\tilde{h}_{r}$ at the location $l_{1}$ which is i.i.d. from the power gain $h_{r}$ at the location $l_{0}$. Let $s=\left(\frac{\tau_{h}-\tau_{\text {hys }}}{P_{T} M_{t} M_{r}}\right)(r+v t)^{\alpha_{r}}$ in (30),

$$
\begin{aligned}
& \operatorname{Pr}\left\{\operatorname{SINR}^{0}(\tilde{r})<\tau_{h}-\tau_{\text {hys }} \mid \alpha_{r}\right\} \\
& =1-\exp \left(-\left(\frac{\tau_{h}-\tau_{\text {hys }}}{P_{T} M_{t} M_{r}}\right) \sigma_{n}^{2}(r+v t)^{\alpha_{r}}\right) \\
& \times \exp \left(-\frac{\lambda \pi^{2}}{\sqrt{3} \sigma} \sum_{l=1}^{4} p_{l} \int_{\alpha} \frac{\left(\frac{\left(\tau_{h}-\tau_{\text {hys }}\right)(r+v t)^{\alpha_{r}} g_{l}}{M_{t} M_{r}}\right)^{\frac{2}{\alpha}} \csc \left(\frac{2 \pi}{\alpha}\right)}{\alpha} d \alpha\right) .
\end{aligned}
$$

Substitute (22) and (37) into (21), the handoff probability with $\varphi=\Theta^{r}$ is given as (23), which completes the proof.

\section{ACKNOWLEDGMENT}

The authors would like to acknowledge the support from the National Key R \& D Program of China with Grant No. YS2017YFGH000842. This research is partially supported by the Royal Society project under Grant IEC170324. The authors thank Prof. Shuguang (Robert) Cui from University of California-Davis to polish and enhance significantly this paper.

\section{REFERENCES}

[1] M. Agiwal, A. Roy, and N. Saxena, "Next generation 5G wireless networks: a comprehensive survey," IEEE Commun. Surveys \& Tutorials, vol. 18, no. 3, pp. 1617-1655, Feb. 2016

[2] J. Shi, L. Lv, Q. Ni, et al., "Modeling and analysis of point-to-multipoint millimeter-wave backhaul networks," IEEE Trans. Wireless Commun., publication online, DOI: 10.1109/TWC.2018.2879109.

[3] W. Roh et al., "Millimeter-wave beamforming as an enabling technology for 5G cellular communications: Theoretical feasibility and prototype results," IEEE Commun. Mag., vol. 52, no. 2, pp. 106-113, Feb. 2014

[4] A. V. Alejos, M. G. Sanchez, and I. Cuinas, "Measurement and analysis of propagation mechanisms at $40 \mathrm{GHz}$ : Viability of site shielding forced by obstacles," IEEE Trans. Veh. Technol., vol. 57, no. 6, pp. 3369-3380, Nov. 2008

[5] T. S. Rappaport, W. Roh and K. Cheun, "Mobile's millimeter-wave makeover," IEEE Spectrum, vol. 51, no. 9, pp. 34-58, Sept. 2014

[6] X. Ge, L. Pan, Q. Li, et al.. "Multi-Path Cooperative Communications Networks for Augmented and Virtual Reality Transmission," IEEE Trans. Multimedia, vol. 19, no. 10, pp. 2345-2358, Oct. 2017

[7] Y. Zhong, X. Ge, H.-H. Yang, et al., "Traffic Matching in 5G Ultra-dense Networks," IEEE Commun. Mag., vol. 56, No.8, pp. 100-105, Aug. 2018

[8] V. Naghshin, M. C. Reed, and N. Aboutorab, "Coverage analysis of packet multi-tier networks with asynchronous slots," IEEE Trans. Commun., vol. 65 , no. 1, pp. 200-215, Jan. 2017

[9] H. S. Dhillon, R. K. Ganti, and J. G. Andrews, "Load-aware modeling and analysis of heterogeneous cellular networks," IEEE Trans. Wireless Commun., vol. 12, no. 4, pp. 1666-1677, Apr. 2013

[10] C. H. Liu and K. L. Fong, "Fundamentals of the downlink green coverage and energy efficiency in heterogeneous networks," IEEE $J$. Select. Areas Commun., vol. 34, no. 12, pp. 3271-3287, Dec. 2016

[11] J. G. Andrews, T. Bai, M. N. Kulkarni, et al., "Modeling and Analyzing Millimeter Wave Cellular Systems," IEEE Trans. on Commun., vol. 65, no. 1, pp. 403-430, Jan. 2017

[12] M. Di Renzo, "Stochastic Geometry Modeling and Analysis of MultiTier Millimeter Wave Cellular Networks," IEEE Trans. on Wireless Commun., vol. 14, no. 9, pp. 5038-5057, Sept. 2015

[13] M. N. Kulkarni, S. Singh, and J. G. Andrews, "Coverage and rate trends in dense urban mmWave cellular networks," in IEEE GLOBECOM, Austin, TX, USA, Dec. 2014, pp. 3809-3814

[14] T. Bai and R. W. Heath, "Coverage and rate analysis for millimeterwave cellular networks," IEEE Trans. Wireless Commun., vol. 14, no. 2, pp. 1100-1114, Feb. 2015

[15] Z. Shi and Q. Zhu, "A calculation method for handoff rate based on angle mobility model," in ICSAI, Yantai, China, May 2012, pp. 1389-1392

[16] A. Talukdar, M. Cudak, and A. Ghosh, "Handoff rates for millimeterwave 5G systems," in IEEE VTC Spring 79th, Seoul, South Korea, May 2014, pp. $1-5$

[17] S. Sadr and R. S. Adve, "Handoff rate and coverage analysis in multitier heterogeneous networks," IEEE Trans. Wireless Commun., vol. 14, no. 5, pp. 2626-2638, May 2015

[18] X. Ge, H. Cheng, G. Mao, et al., "Vehicular communications for 5G cooperative small-cell networks," IEEE Trans. Veh. Technol., vol. 65, no. 10, pp. 7882-7894, Oct. 2016

[19] M. Ekpenyong, J. Isabona, and E. Isong, "Handoffs decision optimization of mobile celular networks," in CSCI, Las Vegas, NV, USA, Dec. 2015, pp. 697-702

[20] X. Zhu, M. Li, W. Xia, et al., "A novel handoff algorithm for hierarchical cellular networks," China Commun., vol. 13, no. 8, pp. 136-147, Aug. 2016

[21] A. Ahmed, L. M. Boulahia, and D. Gaiti, "Enabling vertical handover decisions in heterogeneous wireless networks: a state-of-the-art and a classification," IEEE Commun. Surveys \& Tutorials, vol. 16, no. 2, pp. 776-811, Second Quarter 2014

[22] X. Zhang and J. G. Andrews, "Downlink Cellular Network Analysis With Multi-Slope Path Loss Models," IEEE Trans. Commun., vol. 63, no. 5, pp. 1881-1894, May 2015

[23] S. Sun, G. R. MacCartney and T. S. Rappaport, "A novel millimeterwave channel simulator and applications for $5 \mathrm{G}$ wireless communications," in IEEE ICC, Paris, France, May 2017, pp. 1-7 
[24] S. Sun, T. S. Rappaport, M. Shafi, et al., "Propagation Models and Performance Evaluation for 5G Millimeter-Wave Bands," IEEE Trans. Veh. Technol., vol. 67, no. 9, pp. 8422-8439, Sept. 2018

[25] X. Ge, Y. Qiu, J. Chen, et al., "Wireless fractal cellular networks," IEEE Wireless Commun. Mag., vol. 23, no. 5, pp. 110-119, Oct. 2016

[26] X. Ge, M. Huang, J. Chen, et al., "Wireless single cellular coverage boundary models," IEEE Access, vol. 4, pp. 3569-3577, Jun. 2016

[27] X. Ge, X. Tian, Y. Qiu, et al., "Small Cell Networks with Fractal Coverage Characteristics," IEEE Trans. Commun., vol. 66, no. 11, pp. 5457-5469, Nov. 2018

[28] O. Semiari, W. Saad and M. Bennis, "Joint Millimeter Wave and Microwave Resources Allocation in Cellular Networks with Dual-Mode Base Stations," IEEE Trans. wireless Commun., vol. 16, no. 7, pp. 4802 4816, Jul. 2017

[29] X. Ge, K. Huang, C.-X. Wang, et al., "Capacity analysis of a multi-cell multi-antenna cooperative cellular network with co-channel interference," IEEE Trans. Wireless Commun., vol. 10, no. 10, pp. 3298-3309, Oct. 2011

[30] 3GPP, "TR 38.900, Technical Specification Group Radio Access Network; Study on channel model for frequency spectrum above $6 \mathrm{GHz}$," Jul. 2017

[31] Y. S. Meng, Y. H. Lee and B. C. Ng, "Empirical Near Ground Path Loss Modeling in a Forest at VHF and UHF Bands," IEEE Trans. Antennas Propag., vol. 57, no. 5, pp. 1461-1468, May 2009

[32] T. Bai, R. Vaze and R. W. Heath, "Analysis of Blockage Effects on Urban Cellular Networks," IEEE Trans. Wireless Commun., vol. 13, no. 9 , pp. 5070-5083, Sept. 2014

[33] M. Gapeyenko et al., "Analysis of human-body blockage in urban millimeter-wave cellular communications," IEEE ICC, Kuala Lumpur, May. 2016, pp. 1-7

[34] 3GPP, "TR 36.814, Further advancements for E-UTRA physical layer aspects," Mar. 2017

[35] L. Xiang, X. Ge , C.-X. Wang, et al., "Energy Efficiency Evaluation of Cellular Networks Based on Spatial Distributions of Traffic Load and Power Consumption," IEEE Trans. Wireless Commun., vol. 12, no. 3, pp. 961-973, Mar. 2013

[36] H. S. Dhillon, R. K. Ganti, F. Baccelli and J. G. Andrews, "Modeling and Analysis of K-Tier Downlink Heterogeneous Cellular Networks,"IEEE J. Select. Areas Commun., vol. 30, no. 3, pp. 550-560, April 2012

[37] R. Zi, X. Ge, J. Thompson, et al., "Energy Efficiency Optimization of 5G Radio Frequency Chain Systems," IEEE J. Select. Areas Commun., vol. 34, no. 4, pp. 758-771, April 2016

[38] D. Stoyan, W. Kendall, and J. Mecke, Stochastic Geometry and Its Applications, 2nd ed. John Wiley and Sons, 1996

[39] J. Chen, F. Bin, X. Ge, et al., "A dual-directional path-loss model in 5G wireless fractal small cell networks," in IEEE ICC, Paris, France, May 2017, pp. 1-6

[40] Y. Sun, G. Feng, S. Qin, et al., "The SMART Handoff Policy for Millimeter Wave Heterogeneous Cellular Networks," IEEE Trans. Mobile Comput., vol. 17, no. 6, pp. 1456-1468, 1 Jun. 2018

[41] D. Xenakis, M. Kountouris, L. Merakos, et al., "Performance Analysis of Network-Assisted D2D Discovery in Random Spatial Networks," IEEE Trans. Wireless Commun., vol. 15, no. 8, pp. 5695-5707, Aug. 2016

[42] 3GPP, "TS 36.331, Evolved Universal Terrestrial Radio Access (EUTRA)-Radio Resource Control (RRC) protocol specification,” Apr. 2017

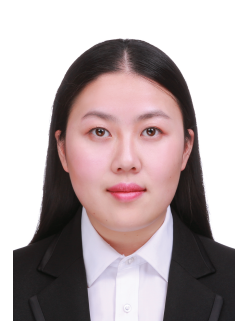

Jiaqi Chen received the B.E. degree in communications engineering from the Huazhong University of Science and Technology, Wuhan, China, in 2013. She is currently working toward the Ph.D. degree with the School of Electronic Information and Communications, Huazhong University of Science and Technology. Her research interests are energy efficiency, fractal cellular networks, and handoff.

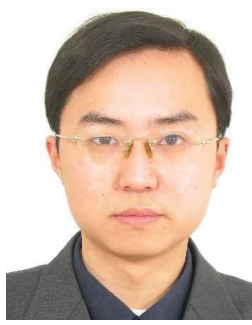

Xiaohu Ge (M'09-SM'11) is currently a full Professor with the School of Electronic Information and Communications at Huazhong University of Science and Technology (HUST), China. He is an adjunct professor with with the Faculty of Engineering and Information Technology at University of Technology Sydney (UTS), Australia. He received his $\mathrm{PhD}$ degree in Communication and Information Engineering from HUST in 2003. He has worked at HUST since Nov. 2005. Prior to that, he worked as a researcher at Ajou University (Korea) and Politecnico Di Torino (Italy) from Jan. 2004 to Oct. 2005. His research interests are in the area of mobile communications, traffic modeling in wireless networks, green communications, and interference modeling in wireless communications. $\mathrm{He}$ has published more than 200 papers in refereed journals and conference proceedings and has been granted about 15 patents in China. He received the Best Paper Awards from IEEE Globecom 2010. Dr. Ge served as the general Chair for the 2015 IEEE International Conference on Green Computing and Communications (IEEE GreenCom 2015). He serves as an associate editor for IEEE Wireless Communications, IEEE Transactions on Vehicular Technology and IEEE ACCESS, etc.

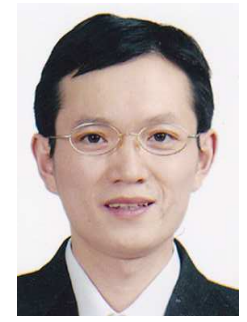

Qiang Ni (M'04-SM'08) received the B.Sc., M.Sc., and Ph.D. degrees from the Huazhong University of Science and Technology, China, all in engineering. $\mathrm{He}$ is currently a Professor and the Head of the Communication Systems Group, School of Computing and Communications, Lancaster University, Lancaster, U.K. His research interests include the area of future generation communications and networking, including green communications and networking, millimeter-wave wireless communication$\mathrm{s}$, cognitive radio network systems, non-orthogonal multiple access (NOMA), heterogeneous networks, 5G and 6G, SDN, cloud networks, energy harvesting, wireless information and power transfer, IoTs, cyber physical systems, machine learning, big data analytics, and vehicular networks. He has authored or co-authored over 200 papers in these areas. $\mathrm{He}$ was an IEEE 802.11 Wireless Standard Working Group Voting Member and a contributor to the IEEE Wireless Standards. 